\title{
On the Fully Commutative Elements of Coxeter Groups
}

\author{
JOHN R. STEMBRIDGE* \\ Department of Mathematics, University of Michigan, Ann Arbor, Michigan 48109-1109
}

Received March 28, 1995; Revised September 21, 1995

\begin{abstract}
Let $W$ be a Coxeter group. We define an element $w \in W$ to be fully commutative if any reduced expression for $w$ can be obtained from any other by means of braid relations that only involve commuting generators. We give several combinatorial characterizations of this property, classify the Coxeter groups with finitely many fully commutative elements, and classify the parabolic quotients whose members are all fully commutative. As applications of the latter, we classify all parabolic quotients with the property that (1) the Bruhat ordering is a lattice, (2) the Bruhat ordering is a distributive lattice, (3) the weak ordering is a distributive lattice, and (4) the weak ordering and Bruhat ordering coincide.
\end{abstract}

Keywords: Coxeter group, reduced word, heap, weak order, Bruhat order

\section{Introduction}

Let $W$ be an arbitrary Coxeter group. This paper is concerned with the elements $w \in W$ with the property that any reduced word for $w$ can be obtained from any other by using only the Coxeter relations that involve commuting generators. We say that such elements are fully commutative.

Our motivation for studying full commutativity arose from some applications we discovered that involve the symmetric functions associated with the Weyl groups of type B and D studied by Billey and Haiman [1], Fomin and Kirillov [8], and Lam [10]. (These applications are discussed in [13].) A second (related) motivation arose from the interesting combinatorial properties of full commutativity in the symmetric group case. For example (quoting [2]), the fully commutative members of $S_{n}$ are the permutations $w$ that avoid the pattern 321 (in one-line notation). The number of these is the Catalan number $C_{n}$, and there is a skew Young diagram $\theta$ naturally associated to each fully commutative $w$ with the property that the standard Young tableaux of shape $\theta$ are in one-to-one correspondence with the reduced words for $w$.

A third motivation, valid in any Coxeter group, is the fact that full commutativity is equivalent to several other natural combinatorial properties. For example (Theorem 3.2 below), $w \in W$ is fully commutative if any only if the set of reduced words for $w$ is ordertheoretic, by which we mean that there is a labeled partially ordered set whose linear extensions are the reduced words for $w$. Also, one can show (again Theorem 3.2) that 
knowledge of the fully commutative elements of $W$ is equivalent to knowledge of the subintervals of the weak ordering of $W$ that are distributive lattices. (By a theorem of Björner [3], one knows that every subinterval of the weak order is at least a lattice.)

In his recent Ph.D. thesis [6] (see also [7]), Fan has independently studied the fully commutative elements of simply-laced ${ }^{1}$ Coxeter groups with an entirely different set of motivations in mind. Fan proves that the fully commutative elements index a basis for a quotient of the associated Iwahori-Hecke algebra. In the symmetric group case, this quotient is the Temperley-Lieb algebra. In the (simply-laced) Weyl group case, Fan gives the following characterization of full commutativity: If $\Phi(w)$ is the set of positive roots sent to negative roots by $w$, then $w$ is fully commutative if and only if the root spaces indexed by $\Phi(w)$ generate an abelian subalgebra of the associated Lie algebra. Fan also uses this characterization as the definition for commutative elements of non simply-laced Weyl groups, but this is not equivalent to full commutativity as we define it.

The outline of the paper is as follows. In Section 3, we prove several characterizations of full commutativity, including the ones mentioned above. Of central importance is the "heap" associated to a fully commutative element $w$-this is a labeled partial order whose linear extensions are the reduced words for $w$. In Section 4, we prove that every fully commutative heap occurs as a convex subset of a heap with unique maximal and minimal elements; these are the heaps of fully commutative double coset representatives of $W$ relative to pairs of maximal parabolic subgroups. We also prove (Theorem 4.4) that a fully commutative element that is maximal with respect to multiplication on the right has a heap with a "top tree" that amounts to a rooted version of the Coxeter graph. In particular, there are no such elements unless the Coxeter graph is acyclic. We then characterize (Theorem 4.5) the rooted trees that arise in this fashion.

In Section 5, we classify the Coxeter groups that are FC-finite (i.e., contain finitely many fully commutative elements). This generalizes the work in [6], where Fan treats the simply-laced case. It is interesting to note that the proof we give is self-contained, purely combinatorial, and close to being a proof of the classification theorem for finite Coxeter groups. (However, there do exist infinite Coxeter groups that are FC-finite.)

In Section 6, we classify the parabolic quotients of Coxeter groups whose members are all fully commutative. The result is that aside from a few exceptional cases, the irreducible quotients with this property arise from orbits of minuscule weights in finite Weyl groups and Coxeter groups in which every edge of the Coxeter graph has infinite weight. Among the finite Weyl groups, this classification coincides with Proctor's classification of the parabolic quotients of Weyl groups whose Bruhat ordering is a lattice [11]. In the final section, we extend Proctor's result by classifying all parabolic quotients of arbitrary Coxeter groups such that (1) the Bruhat ordering is a lattice, (2) the Bruhat ordering is a distributive lattice, (3) the weak ordering is a distributive lattice, and (4) the weak ordering and Bruhat ordering coincide. Interestingly, one finds that all four classification problems have the same answer.

\section{Preliminaries}

Throughout this paper, $W$ shall denote a Coxeter group with finite generating set $S$ and Coxeter matrix $M=[m(s, t)]_{s, t \in S}$. Thus $m(s, t)$ is the order of $s t$ in $W$ (possibly 
$m(s, t)=\infty$ ). We let $\Gamma$ denote the Coxeter graph of $(W, S)$; i.e., the simple graph with vertex set $S$ and edges between pairs of non-commuting generators. By the Coxeter diagram, we mean the pair $(\Gamma, M)$, regarding $M$ as a weight function on the edges of $\Gamma$.

\subsection{Commutativity classes}

Let $S^{*}$ denote the free monoid generated by $S$. We will represent the members of $S^{*}$ as sequences, so that $\mathbf{s}=\left(s_{1}, \ldots, s_{l}\right)$ would be typical. By a subword of $\mathbf{s}$, we shall mean a subsequence of $\mathbf{s}$ occupying consecutive positions, such as $\left(s_{1}, s_{1+1}, \ldots, s_{l}\right)$. Also, for integers $m \geq 0$ and $s, t \in S$, let us define

$$
\langle s, t\rangle_{m}=(\underbrace{s, t, s, t, s, \ldots)}_{m} \in S^{*} .
$$

For $w \in W$, let $\ell(w)$ denote the minimum length of any expression $w=s_{1} \cdots s_{l}$ with $s_{\imath} \in S$. Any such minimum-length expression for $w$ is said to be reduced. It will be convenient more generally to declare any expression of the form $w=w_{1} \cdots w_{l}$ with $w_{i} \in W$ to be reduced if $\ell(w)=\ell\left(w_{1}\right)+\cdots+\ell\left(w_{l}\right)$. We let $\mathcal{R}(w) \subset S^{*}$ denote the set of all words $\mathbf{s}=\left(s_{1}, \ldots, s_{l}\right)$ such that $w=s_{1} \cdots s_{l}$ and the expression is reduced.

Let $\approx$ denote the congruence on $S^{*}$ generated by the braid relations

$$
\langle s, t\rangle_{m(s, t)} \approx\langle t, s\rangle_{m(x, t)}
$$

for all $s, t \in S$ such that $m(s, t)<\infty$. Of central importance for this paper is the fact that if $\mathbf{s}$ is any particular reduced word for $w$, then $\mathcal{R}(w)$ is the equivalence class of $\mathbf{s}$ relative to $\approx$; i.e., any reduced word for $w$ can be obtained from any other by means of the braid relations ([4], Section IV.1.5).

Now consider the weaker congruence $\sim$ on $S^{*}$ generated by the braid relations corresponding to pairs of commuting generators (i.e., the relations $(s, t) \sim(t, s)$ for all $s, t \in S$ such that $m(s, t)=2$ ). We remark that the quotient monoids $S^{*} / \sim$, known in the literature as free partially abelian monoids, or commutation monoids, were first studied in a systematic way by Cartier and Foata [5]. (See also the survey in [14].)

The equivalence class $\mathcal{C}$ of a given reduced word $\mathbf{s}$ (relative to $\sim$ ) consists of the words obtainable from $\mathbf{s}$ by transposing adjacent commuting pairs. We call $\mathcal{C}$ the commutativity class of $\mathbf{s}$. Since $\sim$ is weaker than $\approx$, it is clear that there is a decomposition

$$
\mathcal{R}(w)=\mathcal{C}_{1} \dot{\cup} \ldots \dot{U} \mathcal{C}_{l}
$$

of $\mathcal{R}(w)$ into commutativity classes. If $\mathcal{R}(w)$ consists of just one commutativity class, we say that $w$ is fully commutative.

Proposition 2.1 An element $w \in W$ is fully commutative if and only iffor all $s, t \in S$ such that $3 \leq m(s, t)<\infty$, there is no member of $\mathcal{R}(w)$ that contains $\langle s, t\rangle_{m(s, t)}$ as a subword. 
Proof: Given the fact that any reduced word can be obtained from any other via the braid relations, the sufficiency of the stated condition is clear. To prove that it is also necessary, suppose that $\mathbf{s}$ is a reduced word for some $w \in W$, and that $s, t \in S$ are such that $3 \leq m(s, t)<\infty$. Every member of the commutativity class of $\mathbf{s}$ can be obtained by exchanging adjacent pairs of letters not including the pair $s, t$. It follows that the subsequence of $\mathbf{s}$ formed by the occurrences of $s$ and $t$ is an invariant of the commutativity class of $\mathbf{s}$. Therefore, if $\mathbf{s}$ contains $\langle s, t\rangle_{m}$ as a subword (where $m=m(s, t)$ ), then the reduced word $\mathrm{s}^{\prime}$ obtained by applying the braid relation $\langle s, t\rangle_{m} \approx\langle t, s\rangle_{m}$ belongs to a different commutativity class, and hence $w$ could not be fully commutative.

\subsection{Heaps}

Let $\mathbf{s}=\left(s_{1}, \ldots, s_{l}\right)$ be an arbitrary (i.e., not necessarily reduced) word in $S^{*}$. Define a partial ordering $\preccurlyeq$ on $[l]=\{1,2, \ldots, l\}$ via the transitive closure of the relations

$$
i \prec j \quad \text { if } i<j \text { and } m\left(s_{i}, s_{j}\right) \neq 2 \text {. }
$$

In particular, $i \prec j$ if $i<j$ and $s_{i}=s_{j}$. The triple $P_{\mathbf{s}}=([l], \preccurlyeq, \mathbf{s})$ can be regarded as a labeled poset (i.e., a partial order in which the elements have special labels), the label of the $i$ th vertex being $s_{i}$. Following the terminology of [14], we call $P_{\mathrm{s}}$ the heap of $\mathbf{s}$.

Let $P$ be any partial ordering of $[l]$. By a linear extension of $P$, we mean a total ordering $\pi=(\pi(1), \ldots, \pi(l))$ of $[l]$ consistent with $P$; i.e., $\pi(i)<\pi(j)$ in $P$ implies $i<j$. We let $\mathcal{L}(P)$ denote the set of all linear extensions of $P$. Regarding $\mathbf{s}$ as a labeling of $P$ (i.e., the element $i$ has label $s_{i}$ ), it is convenient to define

$$
\mathcal{L}(P, \mathbf{s})=\left\{\left(s_{\pi(1)}, \ldots, s_{\pi(l)}\right) \in S^{*}: \pi \in \mathcal{L}(P)\right\}
$$

In the case of a heap, the elements with the same label are totally ordered, so there is at most one linear extension corresponding to any given word in $S^{*}$. We will refer to the members of $\mathcal{L}(P, \mathbf{s})$ as labeled linear extensions of $P$.

The following result is a standard part of the theory of heaps (e.g., see Lemma 3.2 of [14] or Exercise 3.48(b) of [12]).

Proposition 2.2 For $\mathrm{s} \in S^{*}, \mathcal{L}\left(P_{\mathrm{s},} \mathrm{s}\right)$ is the commutativity class of $\mathrm{s}$.

Proof: Suppose that $\mathbf{s}^{\prime}=\left(s_{1}^{\prime}, \ldots, s_{l}^{\prime}\right) \in \mathcal{L}\left(P_{\mathbf{s}}, \mathbf{s}\right)$ and that $\pi \in \mathcal{L}\left(P_{\mathbf{s}}\right)$ is the corresponding linear extension. Since adjacent elements in a linear extension must either be incomparable or a covering pair, it follows that for every $k<l$, either $\pi(k)$ and $\pi(k+1)$ are incomparable in $P_{\mathrm{s}}$, or else $s_{k}^{\prime}$ and $s_{k+1}^{\prime}$ do not commute. Therefore, the interchange of any pair of adjacent commuting generators in $\mathbf{s}^{\prime}$ corresponds to the interchange of a pair of adjacent incomparable elements in $\pi$, and hence yields another (labeled) linear extension of $P_{\mathrm{s}}$. Since $\mathbf{s} \in \mathcal{L}\left(P_{\mathrm{s}}, \mathbf{s}\right)$, it follows that $\mathcal{L}\left(P_{\mathrm{s}}, \mathbf{s}\right)$ contains the commutativity class of $\mathbf{s}$.

Conversely, to prove that $\mathcal{L}\left(P_{\mathrm{s}}, \mathrm{s}\right)$ only contains elements from the commutativity class of $\mathbf{s}$, we proceed by induction on the length of $\mathbf{s}$. Suppose that $\pi$ and $\mathbf{s}^{\prime}$ are as above. 
Since $i=\pi(l)$ is a maximal element of $P_{\mathbf{s}}, s_{i}$ must commute with $s_{j}$ for all $j>i$, so $\mathbf{s} \sim \mathbf{s}^{\prime \prime}=\left(s_{1}, \ldots, s_{i-1}, s_{t+1}, \ldots, s_{l}, s_{t}\right)$. However, $\left(s_{1}^{\prime}, \ldots, s_{l-1}^{\prime}\right)$ is a labeled linear extension of the heap of $\left(s_{1}, \ldots, s_{i-1}, s_{l+1}, \ldots, s_{l}\right)$, so by induction we obtain $s^{\prime} \sim s^{\prime \prime}$ and the result follows.

We remark that $P_{\mathbf{s}}$ is an invariant of the commutativity class of $\mathbf{s}$ in the sense that if $\mathbf{s} \sim \mathbf{s}^{\prime}$, then there exists a poset isomorphism $\varphi: P_{\mathbf{s}} \rightarrow P_{\mathbf{s}^{\prime}}$ such that $s_{l}=s_{\varphi(t)}^{\prime}$. In particular, if $w$ is fully commutative, the heaps of the reduced words for $w$ are all equivalent, so we may speak of the heap of $w$ without ambiguity.

\subsection{The weak order}

The (right) weak ordering of $(W, S)$, denoted $\leq_{R}$, is defined to be the transitive closure of the relations $w<{ }_{R} w s$ for all $w \in W, s \in S$ such that $\ell(w)<\ell(w s)$. Equivalently, for all $x, y \in W$ one has $x \leq_{R} x y$ if and only if $x y$ is reduced (i.e., $\ell(x y)=\ell(x)+\ell(y)$ ). The left weak ordering is defined similarly-one has $y \leq_{L} x y$ if and only if $x y$ is reduced. We remark that the map $w \mapsto w^{-1}$ provides an isomorphism between the left and right weak orderings of $W$. Apart from the special case of symmetric groups, the weak ordering of Coxeter groups seems to have been first studied by Björner [3].

Proposition 2.3 For all $x, y \in W$ such that $x \leq_{R} y$, we have

$$
\left\{w \in W: x \leq_{R} w \leq_{R} y\right\} \cong\left\{w \in W: w \leq_{R} x^{-1} y\right\}
$$

as subposets of $\left(W, \leq_{R}\right)$.

Proof: The map $w \mapsto x^{-1} w$ is easily shown to be an isomorphism.

Note that an immediate consequence of Proposition 2.1 is the fact that if $w$ is fully commutative and $w^{\prime} \leq_{R} w$, then $w^{\prime}$ is also fully commutative; i.e.,

Proposition 2.4 The set of fully commutative elements of $W$ forms an order ideal with respect to the right (or left) weak order.

For $w \in W$, let $D_{R}(w)=\{s \in S: \ell(w s)<\ell(w)\}$ and $D_{L}(w)=\{s \in S: \ell(s w)<\ell(w)\}$ denote the right and left descent sets for $w$, respectively. It is well-known (e.g., [9], Section 1.10) that for all $J \subset S$,

$$
\begin{aligned}
& W^{J}=\{w \in W: s \in J \Rightarrow \ell(w s)>\ell(w)\}=\left\{w \in W: D_{R}(w) \cap J=\emptyset\right\} \\
& { }^{J} W=\{w \in W: s \in J \Rightarrow \ell(s w)>\ell(w)\}=\left\{w \in W: D_{L}(w) \cap J=\emptyset\right\}
\end{aligned}
$$

are, respectively, left and right coset representatives for the parabolic subgroup $W_{J}$ generated by $J$. Let us also note that for $I, J \subset S$,

$$
{ }^{I} W^{J}={ }^{I} W \cap W^{J}=\left\{w \in W: D_{L}(w) \cap I=D_{R}(w) \cap J=\emptyset\right\}
$$

forms a set of double coset representatives for $W_{I} \backslash W / W_{J}$. 
Proposition 2.5 For $J \subset S,{ }^{J} W$ (resp., $W^{J}$ ) is an order ideal of the right (resp., left) weak ordering of $W$.

Proof: Let $w \in{ }^{J} W$. If $w^{\prime} \leq_{R} w$, then there exists a reduced expression $w=w^{\prime} x$ for some $x \in W$. Hence for any $s \in J, s w=s w^{\prime} x$ is reduced, so $s w^{\prime}$ is reduced. In other words, $\ell\left(s w^{\prime}\right)>\ell\left(w^{\prime}\right)$ for all $s \in J$, so $w^{\prime} \in{ }^{J} W$.

It should be noted that $W^{J}$ need not be an order ideal of the right weak order.

We remark that if $W$ is finite, with $w_{0} \in W$ being the longest element, the fact that $\ell\left(w_{0} x\right)=\ell\left(x w_{0}\right)=\ell\left(w_{0}\right)-\ell(x)$ for all $x \in W$ (e.g., [9], Section 1.8) shows that $w_{0}$ is the unique maximal element of $W$ with respect to $\leq_{R}$ and $\leq_{L}$. More generally, if $w_{0}^{J} \in W^{J}$ denotes the left coset representative for $w_{0}$, we have the following.

Proposition 2.6 For $J \subset S, w_{0}^{J}$ is the unique maximal element of $\left(W^{J}, \leq_{L}\right)$.

Proof: Let $x_{0}$ denote the longest element of $W_{J}$. Given $w \in W^{J}$, the expression $w x_{0}$ must be reduced (otherwise by the deletion property ([9], Section 5.8) $w$ would not be the shortest member of its coset). Similarly, the expression $w_{0}=w_{0}^{J} x_{0}$ must be reduced. Therefore $w x_{0} \leq_{L} \quad w_{0}=w_{0}^{J} x_{0}$, and hence also $w \leq_{L} w_{0}^{J}$.

\section{Characterizations of full commutativity}

For any partial order $P$, let $J(P)$ denote the distributive lattice of order ideals of $P$.

Lemma 3.1 Let $w \in W$ be of length $l$. If $P$ is a partial order of $[l]$ and $\mathrm{s} \in S^{*}$ is a labeling such that $\mathcal{R}(w)=\mathcal{L}(P, \mathbf{s})$, then $\left\{x \in W: x \leq_{R} w\right\} \cong J(P)$ as posets.

Proof: We claim that for $s \in S, C_{s}:=\left\{i: s_{i}=s\right\}$ is a totally ordered subset of $P$. Indeed, if $i$ and $j$ were incomparable and $s_{i}=s_{J}=s$, then there would exist a linear extension of $P$ in which $i$ and $j$ appear consecutively. However, the corresponding word in $S^{*}$ would have two consecutive occurrences of $s$, and hence could not be a reduced word for $w$, proving the claim.

Now let $s^{(i)}$ denote the $i$ th smallest member of the chain $C_{s}$, relative to $P$. For any $\mathbf{s}^{\prime} \in S^{*}$, define $\nu\left(s, \mathbf{s}^{\prime}\right)$ to be the number of occurrences of $s$ in $\mathbf{s}^{\prime}$.

Suppose that $\mathbf{s}^{\prime}$ is a reduced word for some $x \leq_{R} w$. Since any reduced word for $x$ can be completed to a reduced word for $w$, it follows that $\mathbf{s}^{\prime}$ is an initial segment of some labeled linear extension of $P$, and hence

$$
I\left(\mathrm{~s}^{\prime}\right):=\left\{s^{(l)}: i \leq v\left(s, \mathrm{~s}^{\prime}\right), s \in S\right\}
$$

is an order ideal of $P$. Furthermore, we claim that if $\mathbf{s}^{\prime \prime}$ is another reduced word for $x$, then $I\left(\mathbf{s}^{\prime}\right)=I\left(\mathbf{s}^{\prime \prime}\right)$. If not, then it would be necessary that $v\left(s, \mathbf{s}^{\prime}\right) \neq v\left(s, \mathbf{s}^{\prime \prime}\right)$ for some $s \in S$. Since the suffix of any completion of $\mathbf{s}^{\prime}$ to a reduced word for $w$ can also be used as the suffix for a completion of $s^{\prime \prime}$, it follows that there exist reduced words for $w$ in which the 
multiplicity of $s$ varies. However by assumption, $\mathcal{R}(w)=\mathcal{L}(P, \mathbf{s})$, so every reduced word for $w$ must be a permutation of $\mathbf{s}$.

We can thus use $I(x)$ to denote the common value of $I\left(\mathbf{s}^{\prime}\right)$ for $\mathbf{s}^{\prime} \in \mathcal{R}(x)$. We claim that the map $x \mapsto I(x)$ defines an order-isomorphism between $\left\{x \in W: x \leq_{R} w\right\}$ and $J(P)$.

To prove this, we first note that the map is order-preserving. Indeed, given any covering relation $x<{ }_{R} x s$, we can choose a reduced word for $x$ and complete it to a reduced word for $x s$ by appending $s$, and therefore $I(x) \subset I(x s)$.

To prove that the map is surjective, let $I$ be an order ideal of $P$. One can find $\mathbf{s}^{\prime} \in \mathcal{L}(P, \mathbf{s})$ so that some initial segment of $\mathbf{s}^{\prime}$, say $\mathbf{s}^{\prime \prime}$, is a labeled linear extension of $I$. However by hypothesis, $\mathbf{s}^{\prime}$ must be a reduced word for $w$. Hence $\mathbf{s}^{\prime \prime}$ must be a reduced word for some $x \leq_{R} w$ and $I=I\left(\mathbf{s}^{\prime \prime}\right)=I(x)$.

To prove that the map is injective, suppose that $I(x)=I(y)=I$ for some $x, y \leq_{R} w$. In that case, there must exist a labeled linear extension of $I$ belonging to $\mathcal{R}(x)$. Any completion of this to a labeled linear extension of $P$ (thus yielding a reduced word for $w$ ) must be a reduced word for $x^{-1} w$. On the other hand, since $I(y)=I$, the same argument proves that it must also be a reduced word for $y^{-1} w$, so $x=y$.

Let us declare a subset $\mathcal{R}$ of $S^{*}$ to be order-theoretic if there exists a partial ordering $P$ of [ $l$ ] for some integer $l \geq 0$ and a labeling $\mathbf{s} \in S^{*}$ of $P$ so that $\mathcal{R}=\mathcal{L}(P, \mathbf{s})$.

Theorem 3.2 For $w \in W$, the following are equivalent:

(a) $w$ is fully commutative.

(b) $\left\{x \in W: x \leq_{R} w\right\}$, as a subposet of $\left(W, \leq_{R}\right)$, is a distributive lattice.

(c) $\left\{x \in W: x \leq_{R} w\right\} \cong J\left(P_{\mathbf{s}}\right)$ for some (equivalently, every) $\mathbf{s} \in \mathcal{R}(w)$.

(d) $\mathcal{R}(w)$ is order-theoretic.

(e) $\mathcal{R}(w)=\mathcal{L}\left(P_{\mathbf{s}}, \mathbf{s}\right)$ for some (equivalently, every) $\mathbf{s} \in \mathcal{R}(w)$.

Proof: The implications (c) $\Rightarrow$ (b) and (e) $\Rightarrow$ (d) are immediate, (e) $\Rightarrow$ (c) and (d) $\Rightarrow$ (b) are special cases of Lemma 3.1, and (a) $\Rightarrow$ (e) follows from Proposition 2.2. To complete the proof, it therefore suffices to establish (b) $\Rightarrow$ (a). For this, assume towards a contradiction that $\left\{x \in W: x \leq_{R} w\right\}$ is a distributive lattice, but that $w$ is not fully commutative. Among all such counterexamples, assume that $w$ is one that minimizes length. By Proposition 2.1, there must exist a reduced word $s \in \mathcal{R}(w)$ and a pair $s, t \in S$ such that $\langle s, t\rangle_{m}$ occurs as a subword of $\mathbf{s}$, where $m=m(s, t)$ and $3 \leq m<\infty$. However, Proposition 2.3 shows that if $w^{\prime} \in W$ has a reduced word that occurs as a subword of $\mathbf{s}$, then $\left\{x \in W: x \leq_{R} w^{\prime}\right\}$ is order-isomorphic to a subinterval of $\left\{x \in W: x \leq_{R} w\right\}$. Since subintervals of distributive lattices are also distributive, the minimality of $\ell(w)$ forces $s=\langle s, t\rangle_{m}$; i.e., $w$ must be the longest element of the dihedral Coxeter group generated by $\{s, t\}$. Since the weak ordering of such Coxeter groups is transparently not distributive for $m \geq 3$ (e.g., see figure 1(a) for the case $m=4$ ), we obtain a contradiction.

A subset $C$ of a partial order $P$ is said to be convex if $i, j \in C$ and $i<k<j$ in $P$ implies $k \in C$. The following result provides an intrinsic characterization of the heaps of fully commutative elements. 


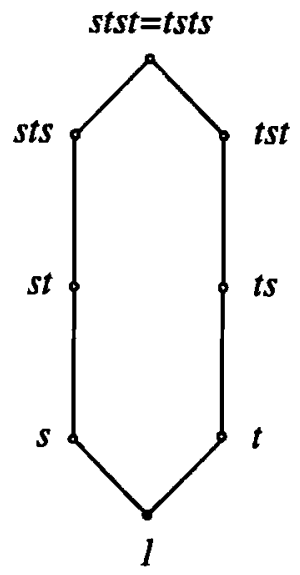

(a)

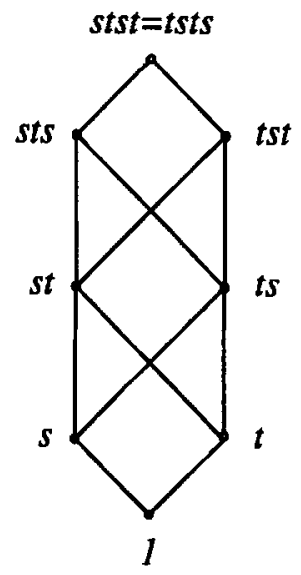

(b)

Figure 1 .

Proposition 3.3 The heap $P$ of a word $\mathbf{s} \in S^{*}$ is the heap of some fully commutative $w \in W$ if and only if

(a) There is no convex chain $i_{1}<\cdots<i_{m}$ in $P$ such that $s_{i_{1}}=s_{i_{3}}=\cdots=s$ and $s_{i_{2}}=s_{t_{4}}=\cdots=t$, where $3 \leq m=m(s, t)<\infty$.

(b) There is no covering relation $i<j$ in $P$ such that $s_{t}=s_{j}$.

Proof: For any convex chain (or covering relation) of a poset $P$, there exist linear extensions in which the members of the chain appear consecutively. Thus if $\mathbf{s}$ is a reduced word for some fully commutative $w \in W$, Proposition 2.1 implies the necessity of (a). Since no reduced word can have two equal adjacent terms, (b) is also necessary. Conversely, given (a), Proposition 2.2 implies that the commutativity class of $\mathbf{s}$ has no members that contain $\langle s, t\rangle_{m}$ as a subword, for all $s, t \in S$ such that $m=m(s, t) \geq 3$. Therefore, the equivalence class of $\mathbf{s}$ relative to the braid relations is the same as its commutativity class. If follows that $P$ is the heap of some fully commutative member of $W$, provided that $\mathbf{s}$ is a reduced word. However, this additional property is a consequence of (b).

\section{Special properties}

Suppose that $P$ is the heap of (a reduced word for) some fully commutative $w \in W$. Recall that for each $s \in S$, the members of $P$ with label $s$ form a chain. It will be convenient for what follows to let $s^{(i)}$ denote the $i$ th greatest member of this chain with respect to $P$. (This is dual to the notation used in the proof of Lemma 3.1, but should not cause confusion.)

Lemma 4.1 Let $s \in S$, and let $w \in W$ be fully commutative with heap $P$. If $w s$ is not fully commutative, then $w s$ is reduced and there is a unique $t \in S$ such that $m(s, t) \geq 3$ 
and $s^{(1)}<t^{(1)}$ in P. Moreover, $m(s, t)<\infty$ and

$$
\begin{aligned}
s^{(k)}<t^{(k)}<s^{(k-1)}<t^{(k-1)}<\cdots<s^{(1)}<t^{(1)} & & (\text { if } m(s, t)=2 k+1) \\
t^{(k)}<s^{(k-1)}<t^{(k-1)}<\cdots<s^{(1)}<t^{(1)} & & (\text { if } m(s, t)=2 k),
\end{aligned}
$$

is a convex chain in $P$.

Proof: Since the fully commutative part of $W$ is an order ideal with respect to $\leq_{R}$ (Proposition 2.4), it follows that if $w s$ is not fully commutative, then $w s$ is reduced. Now let $P_{0}$ be the heap obtained from $P$ by appending $s$ at the end of a reduced word for $w$, and let $s^{(0)}$ denote the new vertex. For $w s$ to not be fully commutative, it is necessary by Proposition 2.3 that for some generator $t \in S$ such that $3 \leq m(s, t)<\infty$, we have

$$
\begin{aligned}
s^{(k)}<t^{(k)} & <\cdots<s^{(1)}<t^{(1)}<s^{(0)} & & \text { (if } m(s, t)=2 k+1) \\
t^{(k)} & <\cdots<s^{(1)}<t^{(1)}<s^{(0)} & & \text { (if } m(s, t)=2 k)
\end{aligned}
$$

occurring as a convex chain in $P_{0}$. If there were another $t^{\prime} \in S$ such that $m\left(s, t^{\prime}\right) \geq 3$ (or $\left.m\left(s, t^{\prime}\right)=\infty\right)$ and $s^{(1)}<\left(t^{\prime}\right)^{(1)}$ in $P$, then we would have $s^{(1)}<\left(t^{\prime}\right)^{(1)}<s^{(0)}$ in $P_{0}$, so the above chain would not be convex.

\subsection{Reduction to maximal quotients}

For $s \in S$, let $\langle s\rangle=S-\{s\}$. Note that the maximal quotient $W^{\langle s\rangle}$ consists of the identity element, together with those $w \in W$ with the property that every $\mathbf{s} \in \mathcal{R}(w)$ ends with $s$. The fully commutative elements with this property are characterized by the fact that their heaps have a maximum element with label $s$.

Theorem 4.2 If $W$ is irreducible and $w \in W$ is fully commutative, then there exists a fully commutative $w^{\prime} \geq_{R} w$ such that $w^{\prime} \in W^{\langle s\rangle}$ for some $s \in S$.

Proof: Let $\mathbf{s}$ be a reduced word for $w$ and $P=P_{\mathbf{s}}$ the heap of $w$. We may assume that every $s \in S$ appears in $\mathbf{s}$, since if $s$ does not appear, then $w s>_{R} w$ and $w s$ is still fully commutative.

Let $D=D_{R}(w) \subset S$ denote the right descent set of $w$. Thus $s \in D$ if and only if $s^{(1)}$ is maximal in $P$. If $D=\{s\}$ is a singleton, then $w \in W^{\langle s\rangle}$ and there is nothing more to prove. Otherwise, let us define the separation of $D$ to be the minimum distance in the Coxeter graph $\Gamma$ among all pairs of elements in $D$. (Note that $\Gamma$ is connected since $W$ is assumed to be irreducible.) We claim that there exists a fully commutative $w^{\prime}>_{R} w$ such that either $\left|D_{R}\left(w^{\prime}\right)\right|<\left|D_{R}(w)\right|$, or else $\left|D_{R}\left(w^{\prime}\right)\right|=\left|D_{R}(w)\right|$ and $D_{R}\left(w^{\prime}\right)$ has a smaller separation than $D_{R}(w)$. By iteration, this result would establish the existence of a fully commutative $w^{\prime}>_{R} w$ such that $\left|D_{R}\left(w^{\prime}\right)\right|=1$, thereby completing the proof.

To prove the claim, consider a pair $s, t \in D$ whose distance in $\Gamma$ is minimal, and let $s=s_{0}, s_{1}, \ldots, s_{l}=t$ be a shortest path from $s$ to $t$. It is necessary that $m\left(s_{t-1}, s_{t}\right) \geq 3$ for $1 \leq i<l, m\left(s_{i}, s_{j}\right)=2$ for $|i-j|>2$ (otherwise the path is not minimal), and $l \geq 2$ 
(otherwise, $s^{(1)}$ and $t^{(1)}$ would be comparable in $P$ and hence could not both be maximal). In particular, since $s_{i-1}$ and $s_{t}$ do not commute, $s_{i-1}^{(1)}$ and $s_{i}^{(1)}$ must be comparable in $P$. Bearing in mind that $s^{(1)}$ and $t^{(1)}$ are both maximal in $P$, it follows that there must exist an index $i$ such that

$$
s_{0}^{(1)}>s_{1}^{(1)}>\cdots>s_{i}^{(1)}<s_{l+1}^{(1)} .
$$

In particular, there are (at least) two elements greater than $s_{i}^{(1)}$ in $P$ whose labels do not commute with $s_{i}$. Thus $w s_{i}$ is reduced and (by Lemma 4.1) fully commutative. Furthermore, in the heap of $w s_{i}$, we have

$$
s_{0}^{(1)}>s_{1}^{(1)}>\cdots>s_{i-1}^{(1)}<s_{i}^{(1)} .
$$

Hence by similar reasoning, $w s_{i} s_{i-1}$ is reduced and fully commutative. Iterating this reasoning, we obtain that $w^{\prime}:=w s_{i} s_{i-1} \cdots s_{1}$ is reduced and fully commutative. Moreover, we have $s=s_{0} \notin D_{R}\left(w^{\prime}\right)$, and there is only one element (namely, $\left.s_{1}\right)$ of $D_{R}\left(w^{\prime}\right)$ not in $D_{R}(w)$, so $\left|D_{R}\left(w^{\prime}\right)\right| \leq\left|D_{R}(w)\right|$. If equality occurs, then we have $s_{1}, t \in D_{R}\left(w^{\prime}\right)$ and the separation of $D_{R}\left(w^{\prime}\right)$ is at most $l-1$.

Let $\leq_{L R}$ denote the partial order on $W$ generated by the union of the left and right weak orders; i.e., the transitive closure of the relations $x<_{L R} x y$ and $y<_{L R} x y$ for all $x, y \in W$ such that $x y$ is reduced. It is clear that the fully commutative elements of $W$ form an order ideal with respect to $<_{L R}$. The following result shows that this order ideal is generated by members of the maximal two-sided quotients of $W$.

Corollary 4.3 If $W$ is irreducible and $w \in W$ is fully commutative, then there exists a fully commutative $w^{\prime} \geq_{L R}$ w such that $w^{\prime} \in{ }^{\langle s\rangle} W^{\langle t\rangle}$ for some $s, t \in S$.

Proof: By Theorem 4.2, there is a fully commutative $w^{\prime} \geq_{R} w$ such that $w^{\prime} \in W^{\langle s\rangle}$ for some $s \in S$. It follows that $s^{(1)}$ is the unique maximal element of the heap of $w^{\prime}$. Without loss of generality, we can assume that every member of $S$ occurs in some (equivalently, every) reduced word for $w^{\prime}$, so that adding elements at the bottom of the heap will not change the fact that $s^{(1)}$ is the unique maximal element of the heap. In other words, for every fully commutative $w^{\prime \prime} \geq_{L} w^{\prime}$, we have $w^{\prime \prime} \in W^{\langle s\rangle}$. However, by the dual version of Theorem 4.2, we can find a fully commutative $w^{\prime \prime} \geq_{L} w^{\prime}$ such that $w^{\prime \prime} \in{ }^{\langle t\rangle} W$ for some $t \in S$, and thus $w^{\prime \prime} \in{ }^{(t)} W \cap W^{(s)}={ }^{(t)} W^{(s)}$.

\subsection{The top tree of a maximal element}

By an ordering of a tree $T$ with vertex set $S$, we mean a partial ordering of $S$ obtained by choosing a special vertex $s_{0} \in S$, and declaring $s<t$ if $t$ is on the unique path from $s$ to $s_{0}$. The Hasse diagram of such an ordering is the tree $T$, rooted at $s_{0}$.

Let $w \in W$ be fully commutative with heap $P$. We will say that $w$ is right-maximal (resp., left-maximal) if for every $s \in S, w s$ (resp., $s w$ ) is either not reduced or not fully 
commutative. In other words, $w$ is maximal in the right (resp., left) weak order with respect to full commutativity. In case $w$ is right-maximal, it will be convenient to define $P^{(1)}=\left\{s^{(1)}: s \in S\right\}$, a subposet of $P$. (We will sometimes abuse this notation and regard $P^{(1)}$ as a partial order on $S$.) It should be noted that every generator must occur in any reduced word for a right-maximal element, so $s^{(1)}$ is indeed defined for all $s \in S$.

The following result explains why we refer to $P^{(1)}$ as the top tre $e^{2}$.

Theorem 4.4 Assume that $W$ is irreducible. If $w \in W$ is fully commutative and rightmaximal with heap $P$, then the Coxeter graph $\Gamma$ is a tree, $P^{(1)}$ is an order filter of $P$, and $P^{(1)}$ is an ordering of the tree $\Gamma$.

Proof: Choose $s \in S$, and suppose that $t^{(l)} \operatorname{covers} s^{(1)}$ in $P$ for some $t \in S$ and $i \geq 1$. It follows that $s^{(1)}<t^{(i)} \leq t^{(1)}$ in $P$ and $m(s, t) \geq 3$. Since $w s$ cannot be fully commutative, Lemma 4.1 implies that the two-element chain $s^{(1)}<t^{(1)}$ must be convex (i.e., a covering relation) in $P$, and therefore $i=1$. In other words, the only members of $P$ that cover $s^{(1)}$ are members of $P^{(1)}$; thus $P^{(1)}$ is an order filter of $P$.

A second consequence of Lemma 4.1 is that there can be at most one element covering $s^{(1)}$ in $P$. Since Theorem 4.2 implies that the heap of a right-maximal $w$ has a unique maximal element, it follows that $P^{(1)}$ is an ordering of some tree. Hence to complete the proof, we must show that this tree is $\Gamma$. Certainly it is true that every covering relation of $P^{(1)}$ must involve a pair of elements whose labels are non-commuting generators--these are the adjacent pairs in $\Gamma$. Conversely, given a pair of non-commuting generators $s, t \in S$, it must be the case that $s^{(1)}$ and $t^{(1)}$ are comparable in $P$; say $s^{(1)}<t^{(1)}$. In that case, since $w s$ cannot be fully commutative, Lemma 4.1 implies that $t^{(1)}$ must be the only element greater than $s^{(1)}$ in $P$ whose label does not commute with $s$, so it must cover $s^{(1)}$.

\subsection{The classification of top trees}

If $Q$ is an ordering of a tree on the vertex set $S$, we will use the notation $t \leftarrow s$ to indicate the covering relation of $Q$; i.e., $t<s$ in $Q$ and $s, t$ are adjacent in the tree.

The following result describes the irreducible Coxeter groups that contain right-maximal fully commutative elements, as well as the top trees of all such elements. Of course by the previous result, we know that $W$ cannot contain any left- or right-maximal elements unless the Coxeter graph $\Gamma$ is a tree, but this is far from sufficient.

Theorem 4.5 Assume that $\Gamma$ is a tree, and let $Q$ be an ordering of $\Gamma$. There exists a fully commutative right-maximal $w \in W$ with top tree $Q$ (i.e., $P^{(1)}=Q$ for the heap $P$ of $w$ ) if and only if the following conditions are satisfied for all $s, t, u \in S$ :

(a) $m(s, t)<\infty$.

(b) If $t \leftarrow s, u \leftarrow s$ and $t \neq u$, then $m(s, t)=m(s, u)=3$.

(c) If $u \leftarrow t \leftarrow s$, then $m(s, t) \leq 4$.

(d) If $u \leftarrow t \leftarrow s$ and $m(s, t)=4$, then $m(t, u)=3$.

Proof: We first prove that conditions (a)-(d) are necessarily satisfied by any right-maximal $w \in W$ with heap $P$ such that $Q=P^{(1)}$. 
(a) If $s, t \in S$ are such that $m(s, t) \geq 3$, then either $s^{(1)}<t^{(1)}$ or $t^{(1)}<s^{(1)}$. Assuming the latter, $w t$ must be reduced and therefore cannot be fully commutative. However by Lemma 4.1, this is possible only if $m(s, t)<\infty$.

(b) Assume towards a contradiction that $t \leftarrow s, u \leftarrow s, t \neq u$, and $m(s, t) \geq 4$. In that case, $w t$ is reduced and therefore cannot be fully commutative. Thus by Lemma 4.1 , $s^{(2)}<t^{(1)}<s^{(1)}$ must occur as a convex chain in $P$. However, $u \leftarrow s$ implies that $u^{(1)}<s^{(1)}$ is a covering relation of $P$. Since $u^{(1)}$ and $s^{(2)}$ must be comparable, we therefore have $s^{(2)}<u^{(1)}<s^{(1)}$, so the chain $s^{(2)}<t^{(1)}<s^{(1)}$ is not convex, a contradiction.

(c) Assume towards a contradiction that $u \leftarrow t \leftarrow s$ and $m(s, t) \geq 5$. As in the previous case, it follows that $w t$ is reduced and therefore cannot be fully commutative. Thus by Lemma $4.1, t^{(2)}<s^{(2)}<t^{(1)}<s^{(1)}$ must occur as a convex chain in $P$. However, $u \leftarrow t$ implies that $u^{(1)}<t^{(1)}$ is a covering relation of $P$. Since $u^{(1)}$ and $t^{(2)}$ must be comparable, it follows that $t^{(2)}<u^{(1)}<t^{(1)}$, so the chain $t^{(2)}<s^{(2)}<t^{(1)}<s^{(1)}$ is not convex, a contradiction.

(d) Assume towards a contradiction that $u \leftarrow t \leftarrow s, m(s, t)=4$, and $m(t, u) \geq 4$. In this case, both $w t$ and $w u$ are reduced and hence neither can be fully commutative. By Lemma 4.1, it follows that both $s^{(2)}<t^{(1)}<s^{(1)}$ and $t^{(2)}<u^{(1)}<t^{(1)}$ must occur as convex chains in $P$. In particular, $s^{(2)}<t^{(1)}$ must be a covering relation. However, $s^{(2)}$ and $t^{(2)}$ must be comparable, so $t^{(2)}<s^{(2)}<t^{(1)}$, contradicting the convexity of the chain $t^{(2)}<u^{(1)}<t^{(1)}$.

For the converse, we assume (a)-(d) and construct a right-maximal $w \in W$ with top tree $Q$, by induction on $|S|$. If $|S|=1$, the nonidentity member of $W$ suffices. If $|S|=2$, then (a) implies that $W$ is a finite dihedral group, and it is straightforward to construct a suitable element $w$ in this case.

Otherwise, we have $|S| \geq 3$. Let $s \in S$ denote the root of $Q$, and let $Q_{1}, \ldots, Q_{k}$ denote the ordered subtrees obtained by deleting the root from $Q$. Each subtree has a root $s_{i} \in S$. Furthermore, the parabolic subgroups $W_{i}$ generated by each $Q_{i}$ commute with each other. By induction, we can find a fully commutative right-maximal element $w_{\iota}$ (relative to $W_{i}$ ) with top tree $Q_{\imath}$, for each $i$.

Case 1. $k \geq 2$, or $k=1$ and $m\left(s_{1}, s\right)=3$. Consider $w=w_{1} \cdots w_{k} s$. Since $s$ does not occur in any reduced expression for $w_{i}$, it is clear that $w$ is fully commutative. To prove that $w$ is right-maximal, choose $t \in S$ and consider $w t$. If $t=s$, then $w t$ is not reduced. If $t$ is an internal vertex of $Q_{i}$, then $w_{i} t$ is reduced and hence cannot be fully commutative, since $w_{i}$ is right-maximal for $W_{i}$. Hence, $w t=w_{1} \cdots\left(w_{i} t\right) \cdots w_{k} s$ cannot be fully commutative. The remaining possibility is that $t=s_{i}$ for some $i$. If $k \geq 2$, then (b) implies $m\left(s_{i}, s\right)=3$; otherwise, if $k=1$ then we have $m\left(s_{i}, s\right)=3$ by hypothesis. Since $s_{i}$ is the root of $Q_{i}$, every reduced word for $w_{i}$ ends with $s_{i}$, so $w_{l} s s_{i}$ is not fully commutative, so $w t=w s_{t}$ is not fully commutative. Thus $w$ is indeed right-maximal, and it is clear that $Q$ is the top tree of $w$.

Case 2. $k=1$ and $m\left(s_{1}, s\right)=4$. (Since $Q_{1}$ has two or more elements, (c) implies that this is the only remaining possibility.) Since $s_{1}$ is the root of $Q_{1}$, there is a reduced expression for $w_{1}$ of the form $w_{1}=w_{1}^{\prime} s_{1}$, where $w_{1}^{\prime} \in W_{1}$. Consider $w=w_{1}^{\prime} s s_{1} s$. 
Since $w_{1}^{\prime} s$ is reduced ( $s$ does not occur in $w_{1}^{\prime}$ ) and $w_{1}^{\prime} s_{1}$ is reduced, it follows that $w_{1}^{\prime}$ is the shortest representative of its left coset, relative to the parabolic subgroup generated by $\left\{s, s_{1}\right\}$. In particular, the expression $w_{1}^{\prime} s s_{1} s$ is reduced.

We claim that $w$ is fully commutative. To see this, we argue incrementally as follows. First, $w_{1}^{\prime} s$ is fully commutative, since $s \notin W_{1}$. Second, $w_{1}^{\prime} s s_{1}$ is fully commutative, since otherwise Lemma 4.1 (and the fact that $m\left(s_{1}, s\right)=4$ ) would imply that some reduced expression for $w_{1}^{\prime}$ must involve $s$. Finally, it follows that $w=w_{1}^{\prime} s s_{1} s$ is fully commutative, since otherwise by Lemma $4.1, s_{1}^{(2)}<s^{(1)}<s_{1}^{(1)}$ must be a convex chain in the heap of $w_{1}^{\prime} s s_{1}$. Hence there would be a reduced expression for $w_{1}^{\prime}$ ending with $s_{1}$, contradicting the fact that $w_{1}^{\prime} s$ is reduced.

Finally, we claim that $w$ is right-maximal. For this, choose $t \in S$ and consider $w t$. If $t=s$, then $w t$ is not reduced. If $t=s_{1}$, then $w t=w_{1}^{\prime} s s_{1} s s_{1}$ is transparently not fully commutative. Otherwise, $t$ is an internal vertex of $Q_{1}$. In particular, it commutes with $s$, and by maximality of $w_{1}, w_{1} t=w_{1}^{\prime} s_{1} t$ is not fully commutative. If $t$ also commutes with $s_{1}$, then $w_{1}^{\prime} t$ must also not be fully commutative, and hence $w t=w_{1}^{\prime} t s s_{1} s$ is not fully commutative. Otherwise, by (d) we have $m\left(s_{1}, t\right)=3$ and Lemma 4.1 implies that there is a reduced expression for $w_{1}^{\prime}$ ending with $t$. Therefore, there is a reduced expression for $w t$ ending with $t s s_{1} s t=s t s_{1} t s$, which is not fully commutative.

\section{The classification of FC-finite Coxeter groups}

We will say that $W$ is $F C$-finite if the number of fully commutative $w \in W$ is finite. The simply-laced FC-finite Coxeter groups were classified by Fan in his thesis [6]; in the following, we treat the general case. It is interesting to note that there are no "exceptional" FC-finite Coxeter groups, in the sense that the irreducible ones occur in seven naturally identifiable infinite families. (See figure 2.)

Theorem 5.1 The irreducible FC-finite Coxeter groups are $A_{n}(n \geq 1), B_{n}(n \geq 2), D_{n}$ $(n \geq 4), E_{n}(n \geq 6), F_{n}(n \cdot \geq 4), H_{n}(n \geq 3)$, and $I_{2}(m)(5 \leq m<\infty)$.

Before beginning the proof, let us outline the strategy. First, we derive a list of necessary conditions that collectively eliminate all Coxeter groups not named in the above list. For the converse, it is well known and easy to show that the groups $A_{n}, B_{n}, D_{n}$ and $I_{2}(m)(m<\infty)$ are finite (and hence, FC-finite), so we confine our attention to proving that the groups $E_{n}$, $F_{n}$ and $H_{n}$ are FC-finite.

Proof: Assume that $W$ is irreducible and FC-finite.

(1) $\Gamma$ must be acyclic. Indeed, suppose that $s_{1}, \ldots, s_{n} \in S$ form a circuit of $\Gamma$, so that $s_{t}$ and $s_{t+1}$ do not commute for $1 \leq i \leq n$ (subscripts taken modulo $n$ ). It follows that any initial segment of the word

$$
\left(s_{1}, s_{2}, \ldots, s_{n}, s_{1}, s_{2}, \ldots, s_{n}, \ldots\right)
$$




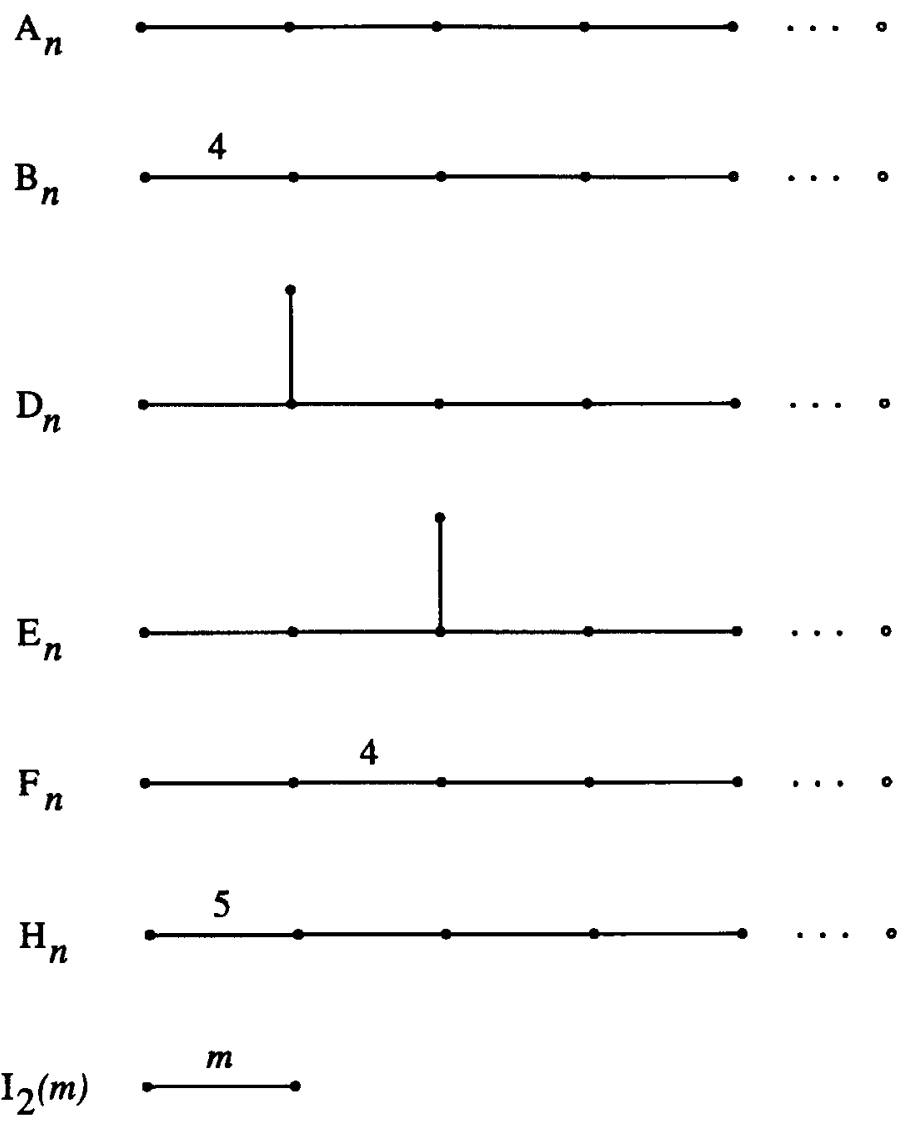

Figure 2. The FC-finite Coxeter groups.

has no subwords of the form $\langle s, t\rangle_{m}$ with $m=m(s, t)$. Hence, any such word is not merely reduced, it is also rigid; i.e., it is the unique reduced word for some $w \in W$. In particular, any such $w$ is fully commutative, so $W$ could not be FC-finite.

(2) Every edge of $\Gamma$ has finite weight. If $m(s, t)=\infty$, then any initial segment of the infinite word $(s, t, s, t, s, t, \ldots)$ is rigid.

(3) $\Gamma$ has at most one edge of weight $\geq 4$. Otherwise, there exists a path $s_{1}, \ldots, s_{n}$ in $\Gamma$ such that $n \geq 3, m\left(s_{1}, s_{2}\right) \geq 4$, and $m\left(s_{n-1}, s_{n}\right) \geq 4$. However in that case, any initial segment of the following infinite word is rigid:

$$
\left(s_{1}, s_{2}, \ldots, s_{n-1}, s_{n}, s_{n-1}, \ldots, s_{2}, s_{1}, s_{2}, \ldots, s_{n-1}, s_{n}, s_{n-1}, \ldots, s_{2}, s_{1}, s_{2}, \ldots\right) .
$$

We remark that an alternative proof of (1) and (2) is provided by the fact that any FC-finite Coxeter group must contain right-maximal fully commutative elements, and hence must satisfy the conditions of Theorems 4.4 and 4.5. We also remark that it is not hard to show that properties (1)-(3) characterize the (irreducible) Coxeter groups with finitely many rigid elements. 
(a)

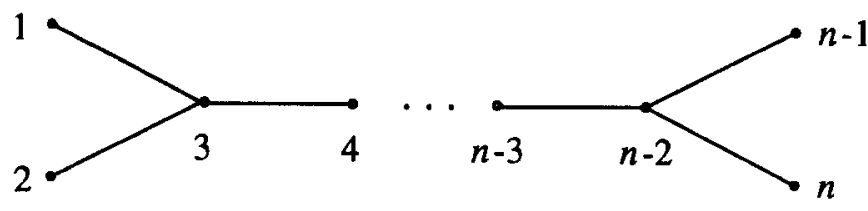

(b)

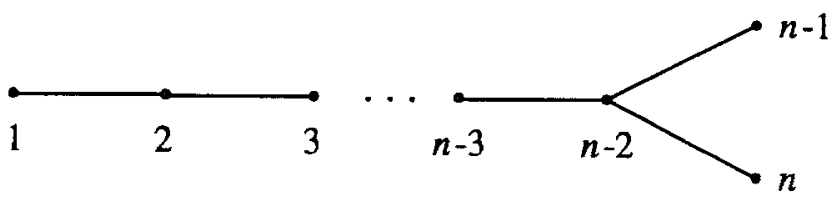

Figure 3.

(4) $\Gamma$ has no vertex of degree $\geq 4$, and at most one vertex of degree 3 . Otherwise, $\Gamma$ contains an induced subgraph isomorphic to the one indicated in figure 3(a); the existence of a vertex of degree 4 corresponds to the case $n=5$. Now consider the infinite word

$$
\left(s_{1}, s_{2}, s_{3}, \ldots, s_{n-2}, s_{n-1}, s_{n}, s_{n-2}, \ldots, s_{3}, s_{1}, s_{2}, s_{3}, \ldots, s_{n-2}, s_{n-1}, s_{n}, s_{n-2}, \ldots\right)
$$

The only subwords of the form $\langle s, t\rangle_{m}$ with $m=m(s, t)$ that occur in this word involve the commuting pairs $\left(s_{1}, s_{2}\right)$ and $\left(s_{n-1}, s_{n}\right)$. Since this property is preserved when any of these pairs are transposed, it follows that every initial segment of this word is reduced and fully commutative.

(5) $\Gamma$ cannot have both a vertex of degree 3 and an edge of weight $\geq 4$. Otherwise, $\Gamma$ contains an induced subgraph isomorphic to the one indicated in figure 3(b), with $m\left(s_{1}, s_{2}\right) \geq 4$ and $n \geq 4$. In this case, consider the infinite word

$$
\left(s_{1}, s_{2}, \ldots, s_{n-2}, s_{n-1}, s_{n}, s_{n-2}, \ldots, s_{2}, s_{1}, s_{2}, \ldots, s_{n-2}, s_{n-1}, s_{n}, s_{n-2}, \ldots\right)
$$

The only subwords of the form $\langle s, t\rangle_{m}$ with $m=m(s, t)$ that occur in this word involve the commuting pair $\left(s_{n-1}, s_{n}\right)$. Since this property is preserved when any of these pairs are transposed, it follows that every initial segment of this word is reduced and fully commutative.

Assuming $W \neq A_{1}$, properties (1)-(5) imply that the Coxeter diagram ( $\left.\Gamma, M\right)$ must be isomorphic to a member of one of the families $Y(p, q, r)$ or $I(p, q ; m)$ indicated in figure 4 , with $p, q, r \geq 1$. Note that in the former case, every edge has weight 3 ; in the latter case, one edge has weight $m$ for some (finite) $m \geq 3$, and the remainder have weight 3 .

(6) If $\max (p, q) \geq 2$ and $m \geq 6$, then $I(p, q ; m)$ is not FC-finite. Indeed, if the generators are labeled so that $m\left(s_{1}, s_{2}\right) \geq 6$ and $m\left(s_{2}, s_{3}\right)=3$, then the infinite word

$$
\left(s_{2}, s_{1}, s_{3}, s_{2}, s_{1}, s_{2}, s_{1}, s_{3}, s_{2}, s_{1}, s_{2}, s_{1}, s_{3}, s_{2}, \ldots\right)
$$




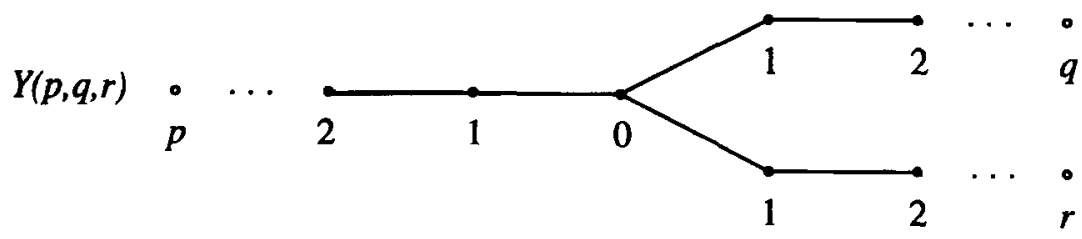

$I(p, q ; m)$

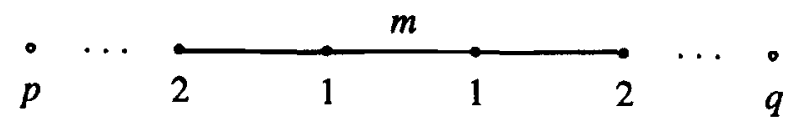

Figure 4.

has the property that the only subwords of the form $\langle s, t\rangle_{m}$ with $m=m(s, t)$ that occur involve the commuting pair $\left(s_{1}, s_{3}\right)$. Furthermore, when any of these pairs are interchanged, the longest alternating $\left(s_{1}, s_{2}\right)$-subword has length 5 , and the occurrences of $\left(s_{2}, s_{3}\right)$ and $\left(s_{3}, s_{2}\right)$ remain disjoint. Hence, any initial segment of this word is reduced and fully commutative. It follows that if $\Gamma$ has an edge of weight $\geq 6$, then $W$ must be one of the (finite) dihedral groups $I_{2}(m)$.

(7) If $p, q \geq 2$, then $I(p, q ; 5)$ is not $F C$-finite. We can assume that the generators are labeled so that $m\left(s_{1}, s_{1}^{\prime}\right)=5$, with $s_{1}, s_{2}, \ldots$ and $s_{1}^{\prime}, s_{2}^{\prime}, \ldots$ forming the two "branches" of the Coxeter graph. Again we claim that there is an infinite word whose initial segments are reduced words for fully commutative members of $W$. However in this case, it is more helpful to describe the heap of this infinite word: See figure 5. (Note that the vertices of the heap have been assigned the labels of the corresponding generators.) One merely needs to check that this poset satisfies the criterion of Proposition 3.3. Once this is done, it follows that every (finite) order ideal of this poset is the heap of some fully commutative element. Thus if the group $W=I(p, q ; 5)$ is FC-finite, it is necessary that $\min (p, q)=1$; however in that case, $W \cong H_{p+q}$.

(8) If $p, q \geq 3$, then $I(p, q ; 4)$ is not $F C$-finite. Let us continue the labeling of the generators established in (7), except that we now have $m\left(s_{1}, s_{1}^{\prime}\right)=4$. In this case, the infinite heap of figure 6 satisfies the conditions of Proposition 3.3, and hence proves that the group in question is not FC-finite. It follows that if $W=I(p, q ; 4)$ is FCfinite, then $\min (p, q)=1$ or 2 . However in that case, $W$ is isomorphic to $B_{p+q}$ or $F_{p+q}$.

The only remaining groups of the form $I(p, q ; m)$ are those for which $m=3$; however, these are Coxeter groups of type A.

(9) If $p, q, r \geq 2$, then $Y(p, q, r)$ is not FC-finite. Let us suppose that the generators are labeled so that $s_{0}$ is the vertex of degree 3 , with $s_{1}, s_{2}, \ldots ; s_{1}^{\prime}, s_{2}^{\prime}, \ldots ;$ and $s_{1}^{\prime \prime}, s_{2}^{\prime \prime}, \ldots$ forming the three branches of $\Gamma$. In this case, the infinite heap of figure 7 proves that these groups cannot be FC-finite.

(10) If $p, q \geq 3$ and $r \geq 1$, then $Y(p, q, r)$ is not $F C$-finite. Continuing the labeling used in (9), the infinite heap of figure 8 proves that these groups cannot be FC-finite. 


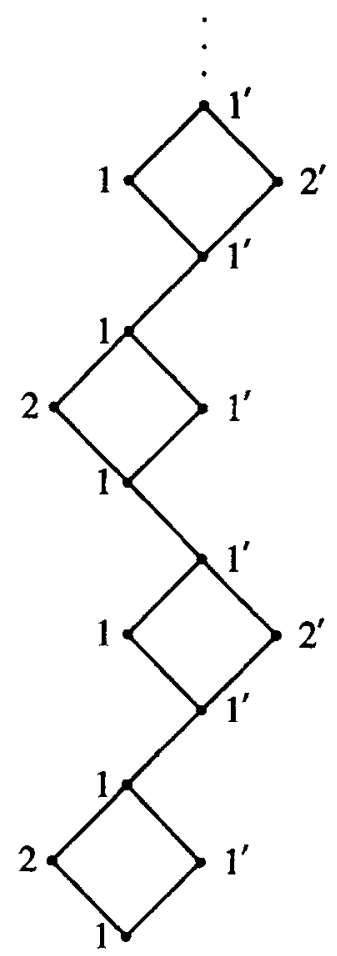

Figure 5.

Properties (9) and (10) prove that if $W=Y(p, q, r)$ is FC-finite and $p \geq q \geq r \geq 1$, then $(q, r)=(1,1)$ or $(q, r)=(2,1)$. However in these respective cases, one has $W \cong D_{p+3}$ and $W \cong E_{p+4}$.

To complete the proof of Theorem 5.1, it remains to be shown that the groups $E_{n}, F_{n}$, and $H_{n}$ are FC-finite. Continuing the notation of Section 4, given a heap $P$ and $s \in S$, let $s^{(i)}$ denote the $i$ th greatest vertex of $P$ with label $s$, relative to the partial order.

Lemma 5.2 Let $W=A_{n}$ and $s \in S$. If $s$ has degree one in $\Gamma$ (or $\left.n=1\right)$, then there is at most one occurrence of $s$ in any reduced word for any fully commutative $w \in W$.

Proof: Let $P$ be the heap of some fully commutative $w \in W$ in which two or more vertices are labeled $s$. Clearly $n \geq 2$, so there is a unique $t \in S$ such that $m(s, t)=3$. It follows that the convex subposet $Q=\left\{j \in P: s^{(2)}<j<s^{(1)}\right\}$ of $P$ is the heap of some fully commutative member $w^{\prime}$ of the parabolic subgroup of type A generated by $S-\{s\}$. Since $Q$ is nonempty (Proposition 3.3(b)), it follows that at least one member of $Q$ covers $s^{(2)}$ and at least one is covered by $s^{(1)}$. The labels of such elements cannot commute with $s$, and hence must be $t$. However by induction with respect to $n$, every reduced word for $w^{\prime}$ has at most one occurrence of $t$. Thus in fact $Q$ must consist of a single vertex with label $t$; given that $w$ is fully commutative, this contradicts Proposition 3.3(a). 


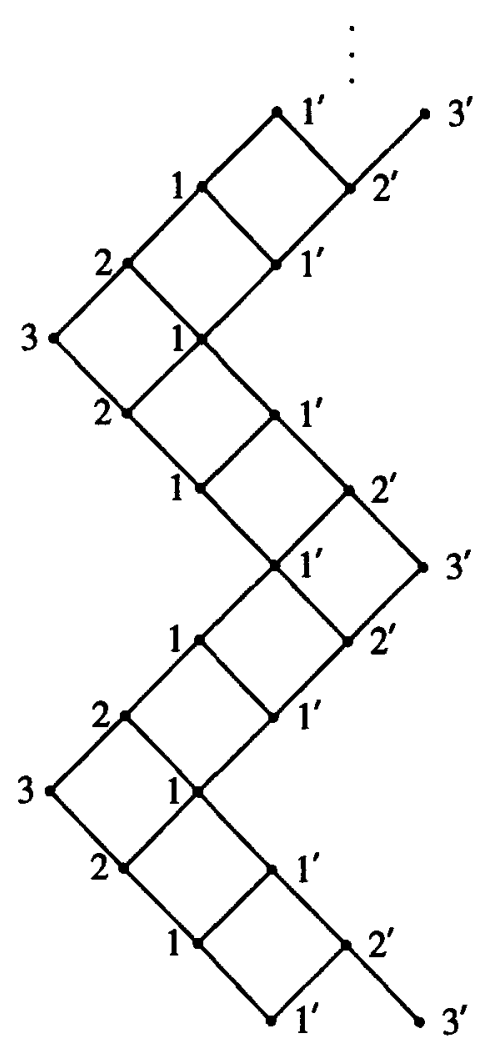

Figure 6.

Suppose that the parabolic subgroup of $W$ generated by some $J \subset S$ is of type A. If there is a unique $s \in J$ and a unique $t \in S-J$ such that $m(s, t) \geq 3$, and if moreover $s$ is an "end node" (i.e., $|J|=1$ or $s$ has degree one relative to $J$ ), then we will say that $J$ is a branch of $S$, with $s$ and $t$ being the points of contact. If $m(s, t)=3$, the branch will be said to be simple.

Lemma 5.3 Let $J$ be a branch of $S$ with points of contact $s \in J$ and $t \in S-J$. If $P$ is the heap of some fully commutative $w \in W$, then for each $i>1$ such that $t^{(i)}$ is defined, there is at most one vertex $j$ of $P$ with label s such that $t^{(i)}<j<t^{(t-1)}$ in $P$. In that case, the chain $t^{(t)}<j<t^{(i-1)}$ is unrefinable.

Proof: Let $t^{(t)}=j_{0}<j_{1}<\cdots<j_{m}=t^{(i-1)}$ be an unrefinable chain of $P$ with at least one member having label $s$. The label sequence corresponding to $j_{0}, \ldots, j_{m}$ must form a path in $\Gamma$ from $t$ to $t$ with no intermediate vertex of label $t$ and at least one vertex with label $s$. Given that $J$ is a branch of $S$, this is possible only if $j_{1}$ and $j_{m}$ both have label $s$. It follows that $Q_{i}=\left\{k \in P: t^{(i)}<k<t^{(i-1)}\right\}$ is the heap of some fully commutative member of $W_{J}$, a Coxeter group of type A. However by Lemma 5.2, any such heap can have at most one vertex with label $s$, so $j_{1}=j_{m}$ and $m=1$. 


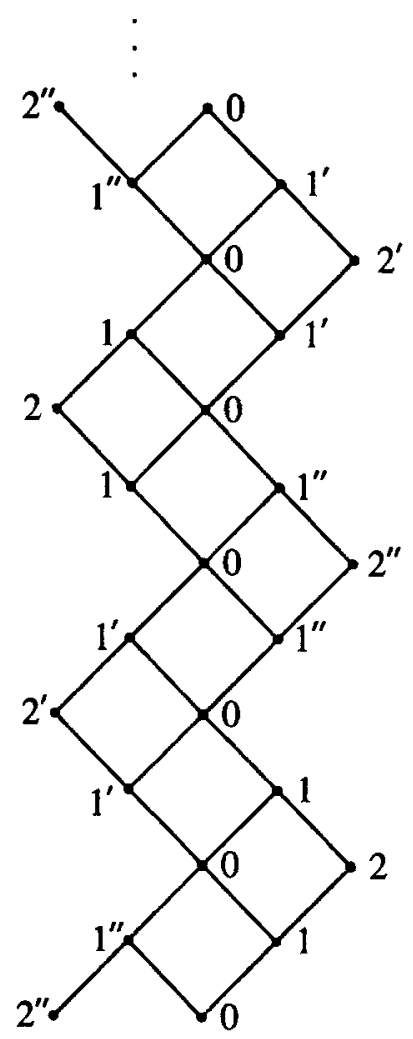

Figure 7.

Lemma 5.4 Let $J$ be a simple branch of $S$ with points of contact $s \in J$ and $t \in S-J$. If $P$ is the heap of some fully commutative $w \in W$ and there is an unrefinable chain $i_{1}<j_{1}<i_{2}<j_{2}<\cdots<i_{m+1}$ in $P$ such that $i_{1}, \ldots, i_{m+1}$ have label $t$ and $j_{1}, \ldots, j_{m}$ have label $s$, then $m \leq|J|$.

Proof: Proceed by induction on $m$, the case $m=1$ being trivial. We note that $J-\{s\}$ is also a simple branch of $S$, with the points of contact being $s$ and some $s^{\prime} \in J-\{s\}$. By Proposition 3.3, the chain $j_{1}<i_{2}<j_{2}$ cannot be convex, so there must exist some other vertex $k$ of $P$ such that $j_{1}<k<j_{2}$, with $k$ covering $j_{1}$. Since $J$ is a branch, the only generators not commuting with $s$ are $t$ and $s^{\prime}$, so this is possible only if the label of $k$ is $s^{\prime}$. However in that case, Lemma 5.3 implies that the chain $j_{1}<k<j_{2}$ is unrefinable. Iterating this argument, we obtain an unrefinable chain $j_{1}<k_{1}<j_{2}<k_{2}<\cdots<j_{m}$ in $P$ such that $k_{1}, \ldots, k_{m-1}$ have label $s^{\prime}$. Hence by the induction hypothesis, we must have $m-1 \leq|J|-1$.

Proof that $\boldsymbol{H}_{n}$ is FC-finite: For $W=H_{n}$, there exist generators $s, t \in S$ such that $m(s, t)=5$ and $S-\{t\}$ is a branch, with the points of contact being $s$ and $t$. Now 


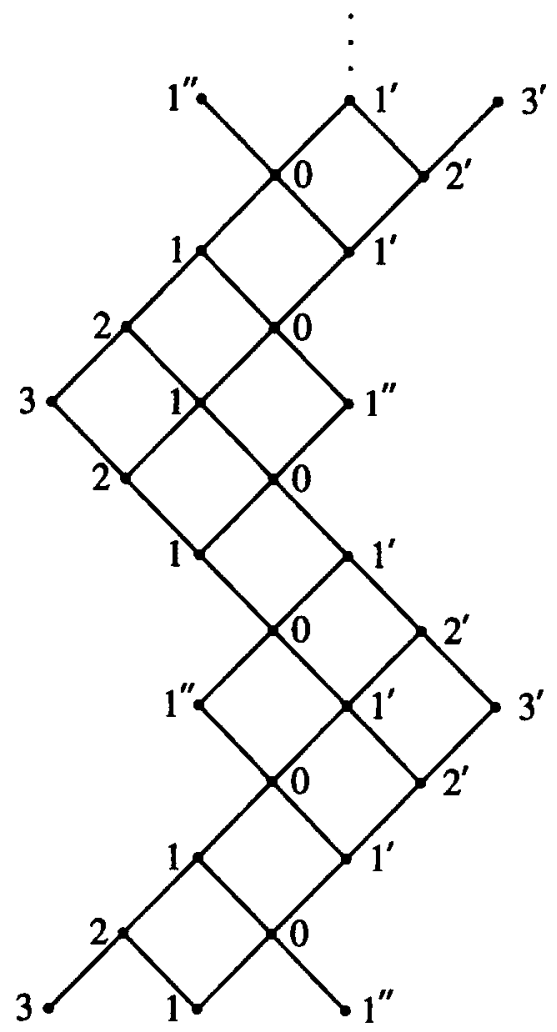

Figure 8.

suppose that $P$ is the heap of some fully commutative $w \in H_{n}$, and let $i_{1}<\cdots<i_{m}$ be the vertices of $P$ with label $t$. Since $s$ is the only generator that does not commute with $t$, Lemma 5.3 implies that there is an unrefinable chain $i_{1}<j_{1}<i_{2}<j_{2}<\cdots<i_{m}$ in $P$ such that $j_{1}, \ldots, j_{m-1}$ have label $s$. Now by Proposition 3.3, $i_{1}<j_{1}<i_{2}<j_{2}<i_{3}$ cannot be a convex chain in $P$. On the other hand, there is a unique $s^{\prime} \in S-\{s, t\}$ that does not commute with $s$ and there is no such vertex that does not commute with $t$. It follows that there must be some vertex $k \in P$ with label $s^{\prime}$ such $j_{1}<k<j_{2}$. Iterating this argument, we obtain the existence of a chain $j_{1}<k_{1}<j_{2}<k_{2}<\cdots<j_{m-1}$ in $P$ in which $k_{1}, \ldots, k_{m-2}$ have label $s^{\prime}$. By Lemma 5.3, this chain must be unrefinable. Furthermore, since $S-\{s, t\}$ is a simple branch of $S$ of size $n-2$, Lemma 5.4 implies that $m-2 \leq n-2$. In other words, every fully commutative $w \in H_{n}$ uses the generator $t$ at most $n$ times. Thus any such element can be expressed in the form $w_{0} t w_{1} t w_{2} \cdots t w_{m}$, where $m \leq n$ and each $w_{i}$ belongs to the (finite) parabolic subgroup generated by $S-\{t\}$.

Proof that $F_{n}$ is FC-finite: Let $s, t \in S$ denote the two generators of $W=F_{n}$ with $m(s, t)=4$, and let $t^{\prime} \in S$ denote the end node with $m\left(t, t^{\prime}\right)=3$ and the property that $\left\{t^{\prime}\right\}$ is a branch of $S$. Now suppose that $P$ is the heap of some fully commutative $w \in F_{n}$. We 
first claim that for each $i$ such that $t^{(i+1)}$ occurs in $P$, there must exist a vertex labeled $s$ in the convex subposet $Q_{i}=\left\{k \in P: t^{(i+1)}<k<t^{(i)}\right\}$. Otherwise, $Q_{i}$ is the heap of some fully commutative $w^{\prime}$ in the parabolic subgroup generated by $S-\{s, t\}$. However, the only member of $S-\{s, t\}$ that does not commute with $t$ is $t^{\prime}$, so by Lemma 5.3, $Q_{i}$ must consist of a singleton vertex with label $t^{\prime}$. This contradicts Proposition 3.3, so the claim follows.

Secondly, we claim that $Q_{i}$ and $Q_{i+1}$ cannot both contain vertices with label $t^{\prime}$. Otherwise, there would exist a chain $t^{(i+2)}<k_{1}<t^{(i+1)}<k_{2}<t^{(t)}$, necessarily unrefinable (Lemma 5.3), in which $k_{1}$ and $k_{2}$ both have label $t^{\prime}$. However, $\left\{t^{\prime}\right\}$ is a simple branch of $S$, so this contradicts Lemma 5.4 .

Let $i_{1}<\cdots<i_{m}$ denote the vertices of $P$ with label $t$. By the first claim, there is a chain $i_{1}<j_{1}<i_{2}<j_{2}<\cdots<i_{m}$ in $P$ such that $j_{1}, \ldots, j_{m-1}$ have label $s$. By Lemma 5.3, this chain must be unrefinable. By the second claim, there is either no vertex $k$ of $P$ with label $t^{\prime}$ such that $i_{1}<k<i_{2}$ or else no such vertex with $i_{2}<k<i_{3}$. If the former holds, consider the chain $i_{1}<j_{1}<i_{2}<j_{2}$; if the latter, consider $j_{1}<i_{2}<j_{2}<i_{3}$. By Proposition 3.3 , neither chain can be convex. Since every generator commutes with either $s$ or $t$, and we have eliminated the possibility of a vertex labeled $t^{\prime}$ between $i_{1}$ and $i_{2}$ (the former case) or between $i_{2}$ and $i_{3}$ (the latter case), the only remaining possibility is that there is a vertex $k$ such that $j_{1}<k<j_{2}$, with the label of $k$ being a generator not commuting with $s$, other than $t$. Note there is a unique generator, say $s^{\prime}$, with this property. Note also that $S-\left\{s, t, t^{\prime}\right\}$ is a simple branch of $S$, with the points of contact being $s$ and $s^{\prime}$.

By iterating this argument, we obtain a chain $j_{1}<k_{1}<j_{2}<k_{2}<\cdots<j_{m-1}$ in $P$ with the property that $k_{1}, \ldots, k_{m-2}$ have label $s^{\prime}$. By Lemma 5.3, this chain must be unrefinable. Furthermore, since $S-\left\{s, t, t^{\prime}\right\}$ is a simple branch, Lemma 5.4 implies that $m-2 \leq n-3$. In other words, every fully commutative $w \in F_{n}$ uses the generator $t$ at most $n-1$ times. Thus any such element can be expressed in the form $w_{0} t w_{1} t w_{2} \cdots t w_{m}$, where $m<n$ and each $w_{t}$ belongs to the (finite) parabolic subgroup generated by $S-\{t\}$.

Proof that $\boldsymbol{E}_{\boldsymbol{n}}$ is FC-finite: We can label the generators of $W=E_{n}$ so that $t$ has degree 3 in $\Gamma$, and $s, s^{\prime}, s^{\prime \prime}$ are the generators adjacent to $t$. We can arrange the labels so that there are (simple) branches of sizes $n-4,2$ and 1 , with points of contact $t$ and (respectively) $s$, $s^{\prime}$ and $s^{\prime \prime}$. Now suppose that $P$ is the heap of some fully commutative $w \in E_{n}$. Assuming that $t^{(i+1)}$ occurs in $P$, consider the convex subposet $Q_{i}=\left\{k \in P: t^{(i+1)}<k<t^{(i)}\right\}$ of $P$. The possible labels of elements covering $t^{(i+1)}$ are $s, s^{\prime}, s^{\prime \prime}$. Since each of them is a point of contact for a branch at $t$, Lemma 5.3 implies that each such element must also be covered by $t^{(l)}$. Since Proposition 3.3 implies that $Q_{t}$ cannot be a singleton, there are three remaining possibilities:

(a) $Q_{t}$ is a tripleton, with vertices labeled $s, s^{\prime}$, and $s^{\prime \prime}$.

(b) $Q_{i}$ is a doubleton, with vertices labeled $s$ and $s^{\prime}$.

(c) $Q_{i}$ is a doubleton, with vertices labeled $s$ and $s^{\prime \prime}$.

(d) $Q_{i}$ is a doubleton, with vertices labeled $s^{\prime}$ and $s^{\prime \prime}$.

Note that the members of $Q_{i}$ are incomparable in $P$.

Given that there are $m$ occurrences of the label $t$ in $P$, we can construct a word $\alpha$ of length $m-1$ in the alphabet $\{a, b, c, d\}$, according to the type of each subinterval $Q_{1}, \ldots, Q_{m-1}$. 
Since $\left\{s^{\prime \prime}\right\}$ is a simple branch of $S$, Lemma 5.4 implies that there can be no subword of $\alpha$ of the form $x y$, where $x, y \in\{a, c, d\}$. Furthermore, we claim that the letter $d$ can appear only at the beginning or end of $\alpha$. Otherwise, the only possible subword of the form $x d y$ that avoids the previously forbidden subwords of length 2 is $b d b$. However if this occurs, then for some $i$, each of $Q_{i}, Q_{i+1}, Q_{i+2}$ contain vertices labeled $s^{\prime}$, contradicting Lemma 5.4 and the fact that there is a simple branch of size 2 connecting $s^{\prime}$ and $t$.

Since $d$ is the only interval-type omitting vertices labeled $s$, it follows that $Q_{2}, \ldots, Q_{m-2}$ must each contain a vertex labeled $s$. Since there is a simple branch of size $n-4$ connecting $s$ and $t$, Lemma 5.4 implies that $m-3 \leq n-4$. In other words, the generator $t$ can appear at most $n-1$ times in any fully commutative $w \in E_{n}$. Thus any such $w$ can be expressed in the form $w_{0} t w_{1} t w_{2} \cdots t w_{m}$, where $m<n$ and each $w_{i}$ belongs to the (finite) parabolic subgroup generated by $S-\{t\}$.

\section{Fully commutative quotients}

By Theorem 4.2, we know that the order ideal (with respect to $\leq_{R}$ ) of fully commutative elements of $W$ is generated by the fully commutative parts of the maximal parabolic quotients $W^{\langle s\rangle}$ for $s \in S$. Thus to a large extent, the task of determining all fully commutative elements of $W$ reduces to the corresponding question for maximal quotients. In the case of the symmetric groups, the situation is particularly simple, since it is known (and it also follows from what will be demonstrated below) that every member of every maximal quotient is fully commutative. This raises the question: Which parabolic quotients of Coxeter groups (not necessarily maximal) have the property that every member is fully commutative? As we shall see, apart from degenerate cases, the answer to this question also turns out to be the answer to several very natural order-theoretic questions about parabolic quotients.

Let $J \subset S$. The quotient $W^{J}$ will be said to be minuscule if $W$ is (isomorphic to) a finite Weyl group and the subgroup $W_{J}$ is the stabilizer of a minuscule weight $\lambda$. (A nonzero weight $\lambda$ is minuscule if there is a representation of a semisimple Lie algebra with Weyl group $W$ whose set of weights is the $W$-orbit of $\lambda$.) The classification of minuscule weights is well-known and can be found in Exercise VI.4.15 of [4], for example. Assuming that $W$ is irreducible, the pairs $\left(W, W_{J}\right)$ such that the quotient $W^{J}$ is minuscule are as follows:

1. $W \cong A_{n} ; J=S-\{s\}$ for any $s \in S$.

2. $W \cong B_{n} ; W_{J} \cong B_{n-1}$ or $A_{n-1}$.

3. $W \cong D_{n} ; W_{J} \cong D_{n-1}$ or $A_{n-1}$.

4. $\left(W, W_{J}\right) \cong\left(E_{6}, D_{5}\right)$ or $\left(E_{7}, E_{6}\right)$.

Note that all irreducible minuscule quotients are also maximal quotients.

Theorem 6.1 Assume that $W$ is irreducible. If $J$ is a proper subset of $S$, then every member of $W^{J}$ is fully commutative if and only if one of the following is true:

(a) Every edge of $\Gamma$ has infinite weight (i.e., $m(s, t) \geq 3 \Rightarrow m(s, t)=\infty$ ).

(b) $W^{J}$ is minuscule.

(c) $\left(W, W_{J}\right) \cong\left(H_{3}, I_{2}(5)\right)$ or $\left(I_{2}(m), A_{1}\right)$. 


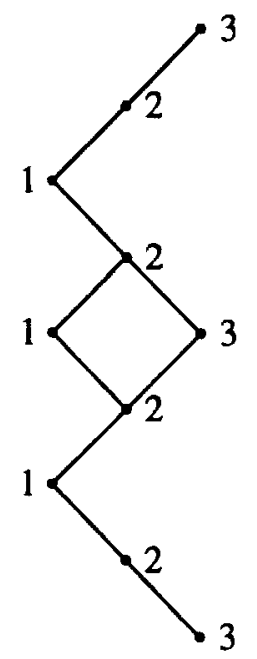

Figure 9.

Proof: First, we show that properties (a)-(c) are each sufficient to imply that every member of $W^{J}$ is fully commutative. Indeed, if (a) holds, then the only braid relations involve pairs of commuting generators, and hence every member of $W$ is fully commutative. If $W=I_{2}(m)$, then there is only one member of $W$ that is not fully commutative; namely, the longest element $w_{0}$. It has (right) descent set $S$, and hence does not belong to $W^{J}$ unless $J=\emptyset$.

In case $W \cong H_{3}$, label the generators $s_{1}, s_{2}, s_{3}$ so that $m\left(s_{1}, s_{2}\right)=5$ and $m\left(s_{2}, s_{3}\right)=3$. Also, set $J=\left\{s_{1}, s_{2}\right\}$, so that $W_{J} \cong I_{2}(5)$. Now consider the heap $P$ of

$$
\mathbf{s}=\left(s_{3}, s_{2}, s_{1}, s_{2}, s_{3}, s_{1}, s_{2}, s_{1}, s_{2}, s_{3}\right)
$$

depicted in figure 9. Using only Proposition 3.3, it is clear that $P$ is the heap of some fully commutative $w \in W$. Furthermore, since the unique maximal element of $P$ has label 3, we have $w \in W^{J}$. Bearing in mind that the longest elements of $H_{3}$ and $I_{2}(5)$ have respective lengths 15 and 5 , it follows that $w$ must be $w_{0}^{J}$ (the longest element of $W^{J}$ ), since it has length $15-5=10$. However, $w_{0}^{J}$ is the unique maximal element of $W^{J}$ with respect to $\leq_{L}$ (Proposition 2.6), so every member of $W^{J}$ is fully commutative (Proposition 2.5).

Now consider (b); i.e., we suppose that $W$ is a finite Weyl group with a crystallographic root system $\Phi$ embedded in some real Euclidean space $\mathbf{E}$ with inner product $(\cdot, \cdot)$, simple roots $\Delta \subset \Phi$, weight lattice $\Lambda \subset \mathbf{E}$, and $W_{J}$ is the stabilizer of some minuscule weight $\lambda \in \Lambda$. For each $\alpha \in \Phi$, let $s_{\alpha} \in W$ denote the reflection on $\mathbf{E}$ fixing the hyperplane perpendicular to $\alpha$, so that $S=\left\{s_{\alpha}: \alpha \in \Delta\right\}$.

Temporarily, let us discard the hypothesis that $\lambda$ is minuscule, and instead merely assume that $\lambda \in \mathbf{E}$ belongs to the closure of the fundamental chamber (i.e., $(\lambda, \alpha) \geq 0$ for all $\alpha \in \Delta$ ). In this case, one knows (e.g., [9], Section 1.12) that the stabilizer of $\lambda$ is $W_{J}$, where $J=\left\{s_{\alpha} \in S:(\lambda, \alpha)=0\right\}$. 
Lemma 6.2 Given $w \in W^{J}$ and $\alpha \in \Delta$, we have $s_{\alpha} w<{ }_{L} w$ if and only if $(w \lambda, \alpha)<0$.

Proof: If $s_{\alpha} w<_{L} w$, then it is necessarily the case that $s_{\alpha} w \lambda \neq w \lambda$; otherwise, $s_{\alpha} w$ would be a member of $w W_{J}$, contradicting the fact that $w$ is the shortest member of its coset. Hence, $(w \lambda, \alpha) \neq 0$. However since $\ell\left(s_{\alpha} w\right)<\ell(w)$, it follows that $w^{-1} \alpha$ must be a negative root (e.g., [9], Section 1.6). On the other hand, $\lambda$ is in the closure of the fundamental chamber, so we must have $(w \lambda, \alpha)=\left(\lambda, w^{-1} \alpha\right)<0$. Conversely, if $\left(\lambda, w^{-1} \alpha\right)<0$, then $w^{-1} \alpha$ is a negative root, so $\ell\left(s_{\alpha} w\right)<\ell(w)$ (again [9], Section 1.6), so $s_{\alpha} w<_{L} w$.

Now suppose that $w \in W^{J}$ is not fully commutative. By replacing $w$ with some $w^{\prime}<_{L} w$ if necessary (cf. Propositions 2.1 and 2.5), we can assume that $w$ has a reduced expression of the form $x_{0} y$, where $x_{0}$ is the element of length $m(s, t)$ in the parabolic subgroup generated by some $\{s, t\} \subset S$ such that $m(s, t) \geq 3$. Now let $\alpha, \beta \in \Delta$ denote the simple roots corresponding to $s$ and $t$, so that $s=s_{\alpha}$ and $t=s_{\beta}$. Since $\ell\left(s x_{0}\right)=\ell\left(t x_{0}\right)<\ell\left(x_{0}\right)$, it follows that $s w, t w<_{L} w$, and hence by Lemma $6.2,(w \lambda, \alpha)<0$ and $(w \lambda, \beta)<0$.

For $\gamma \in \Phi$, let $\gamma^{\vee}=2 \gamma /(\gamma, \gamma)$ denote the corresponding co-root. Since $\Phi$ is assumed to be crystallographic, it follows that $\left(w \lambda, \alpha^{\vee}\right)$ and $\left(w \lambda, \beta^{\vee}\right)$ are negative integers. Furthermore, since $s$ and $t$ generate an irreducible Weyl group of rank 2, it follows that there is at least one root in the positive integral span of $\alpha$ and $\beta$, and the same is true of $\alpha^{\vee}$ and $\beta^{\vee}$ relative to the co-root system $\Phi^{\vee}$. That is, there exist integers $c_{1}, c_{2}>0$ and $\gamma \in \Phi$ such that $\gamma^{\vee}=c_{1} \alpha^{\vee}+c_{2} \beta^{\vee}$. Thus we obtain

$$
\left(w \lambda, \gamma^{\vee}\right)=c_{1}\left(w \lambda, \alpha^{\vee}\right)+c_{2}\left(w \lambda, \beta^{\vee}\right) \leq-c_{1}-c_{2} \leq-2 .
$$

However, by Exercise VI.1.24 of Bourbaki [4], one knows that if $\lambda$ is minuscule, then $\left(w \lambda, \gamma^{\vee}\right) \in\{0, \pm 1\}$ for all $\gamma \in \Phi$ and $w \in W$. This contradicts the hypothesis that $W^{J}$ contains elements that are not fully commutative.

We remark that in Proposition 10 of [6], Fan gives a different proof that every member of a simply-laced minuscule quotient is fully commutative.

Turning to the converse, we derive a series of conditions that are necessary for every member of $W^{J}$ to be fully commutative, and we show that these conditions collectively eliminate all parabolic quotients other than those listed in (a)-(c). To begin with, we will assume that $\Gamma$ has at least one edge of finite weight (since otherwise (a) applies).

(1) Every edge of $\Gamma$ has finite weight. Otherwise, given $s \in S-J$, there are three possibilities: (i) there is a pair $t, u \in S$ such that $m(s, t)=\infty$ and $3 \leq m(s, u)<\infty$, or there is a path $s_{1}, s_{2}, \ldots, s_{n}=s$ in $\Gamma$ such that (ii) $m\left(s_{1}, s_{2}\right)=\infty$ and $m\left(s_{2}, s_{3}\right)=m<\infty$, or (iii) $m\left(s_{1}, s_{2}\right)=m<\infty$ and $m\left(s_{2}, s_{3}\right)=\infty$. In these respective cases, we claim that the following are reduced words for some member of $W^{J}$ that is not fully commutative:

(i) $\mathrm{s}=\left(\langle s, u\rangle_{m}, t, s\right)$, where $m=m(s, u)$.

(ii) $\mathbf{s}=\left(\left\langle s_{2}, s_{3}\right\rangle_{m}, s_{1}, s_{2}, \ldots, s_{n}\right)$.

(iii) $\mathbf{s}=\left(\left\langle s_{1}, s_{2}\right\rangle_{m}, s_{3}, s_{2}, s_{3}, \ldots, s_{n}\right)$.

In each case, it is straightforward to check that $s$ is indeed a reduced word for some $w \in W$. In fact, one finds that $\mathcal{R}(w)$ has either two or three members, depending on 
whether $t$ and $u$ commute (case (i)) or $s_{1}$ and $s_{3}$ commute (cases (ii) and (iii)). It is also transparent that $w$ is not fully commutative. Since each of the two or three members of the braid equivalence class of $s$ ends with $s$, it follows that $w \in W^{J}$ and the claim follows.

(2) $W^{J}$ is a maximal quotient. Otherwise, let $s=s_{1}, \ldots, s_{n}=t$ be a path in $\Gamma$ that connects $s, t \in S-J$, and consider the word

$$
\mathbf{s}=\left(\left\langle s_{1}, s_{2}\right\rangle_{m}, s_{3}, \ldots, s_{n}\right),
$$

where $m=m\left(s_{1}, s_{2}\right)$. (We may assume $m<\infty$, by (1).) It is easy to see that $\mathbf{s}$ is a reduced word for some $w \in W$ that is not fully commutative. Furthermore, every member of the braid equivalence class of $s$ ends with either $t=s_{n}$ or $s=s_{1}$, and the latter occurs if and only if $s_{1}$ commutes with $s_{3}, \ldots, s_{n}$. Hence $D_{R}(w) \subset\{s, t\}$ and $w \in W^{J}$.

Henceforth, we may assume that $J=S-\{s\}$ for some fixed $s \in S$. In this situation, we have $w \in W^{J}$ if and only if $D_{R}(w)=\{s\}$ or $w=1$. Thus we can reformulate our objective as one of identifying conditions that force the existence of $w \in W$ with $D_{R}(w)=\{s\}$ that are not fully commutative.

Lemma 6.3 Let $I \subset S-\{s\}$, and suppose there is a path in $\Gamma$ from some $t \in I$ to $s$ that meets I only at $t$. If there is some $w \in W_{I}$ with $D_{R}(w)=\{t\}$ that is not fully commutative, then there is some $w^{\prime} \in W$ with $D_{R}\left(w^{\prime}\right)=\{s\}$ that is not fully commutative.

Proof: Let $t=s_{1}, \ldots, s_{n}=s$ be the given path in $\Gamma$, and suppose that $w \in W_{I}$ is not fully commutative and $D_{R}(w)=\{t\}$. Consider $w^{\prime}=w s_{2} s_{\mathfrak{Z}} \cdots s_{n}$. Every reduced word for $w$ ends with $t$. Furthermore, since $s_{2}, \ldots, s_{n}$ do not appear in $w$ and $s_{i}$ does not commute with $s_{i+1}$, it follows that the expression $w s_{2} s_{3} \cdots s_{n}$ is reduced, and every reduced word for $w^{\prime}$ consists of a reduced word for $w$ followed by $\left(s_{2}, \ldots, s_{n}\right)$. Therefore $w^{\prime}$ is not fully commutative and $D_{R}\left(w^{\prime}\right)=\{s\}$.

(3) $\Gamma$ is acyclic. If not, then by Lemma 6.3 we can assume that there is a circuit of $\Gamma$ through $s$. Assuming that $s_{1}, \ldots, s_{n}=s$ are the vertices of a minimal circuit, so that $s_{t}$ and $s_{t+1}$ do not commute for $1 \leq i \leq n$ (subscripts taken modulo $n$ ), consider the word $s$ of $(*)$ and the corresponding $w \in W$. In (2), we noted that $D_{R}(w) \subset\left\{s_{1}, s_{n}\right\}$, and that $s_{1} \in D_{R}(w)$ if and only if $s_{1}$ commutes with $s_{3}, \ldots, s_{n}$. However in this case, $s_{1}$ and $s_{n}$ do not commute, so $D_{R}(w)=\left\{s_{n}\right\}=\{s\}$.

(4) $\Gamma$ has no vertex of degree $\geq 4$, and at most one vertex of degree 3 . Otherwise, by following a path from $s$ to a vertex of degree $\geq 3$, we can use Lemma 6.3 to reduce to a configuration in which there are generators $s_{1}, \ldots, s_{n} \in S$ that induce a subgraph of $\Gamma$ isomorphic to the one in figure 3(b), with $s=s_{2}$. (The case $n=4$ occurs when there is a vertex of degree $\geq 4$.) In that case, consider

$$
\mathbf{s}=\left(\left\langle s_{1}, s_{2}\right\rangle_{m}, s_{3}, \ldots, s_{n-2}, s_{n-1}, s_{n}, s_{n-2}, \ldots, s_{3}, s_{2}\right)
$$


where $m=m\left(s_{1}, s_{2}\right)$. By examining the heap of $\mathbf{s}$ and the equivalent word obtained by applying the braid relation $\left\langle s_{2}, s_{1}\right\rangle_{m} \approx\left\langle s_{2}, s_{1}\right\rangle_{m}$, one can see that $s$ is a reduced word for some $w \in W$ that is not fully commutative. Both heaps have a maximum element with label $s=s_{2}$, so $D_{R}(w)=\{s\}$.

(5) Either $W$ is of type $A$, or $s$ is an end node. If $\Gamma$ has a vertex of degree 3 and $s$ has degree $\geq 2$, then there is a configuration in $\Gamma$ isomorphic to the one in (4). Otherwise, $\Gamma$ is a path. Assuming $W \neq A_{n}$ and that $s$ has degree 2, it follows that there is a path $s_{1}, \ldots, s_{n}$ in $\Gamma$ such that $n \geq 3, m\left(s_{n-1}, s_{n}\right) \geq 4$, and $s=s_{2}$. In that case, consider

$$
\mathbf{s}=\left(\left\langle s_{1}, s_{2}\right\rangle_{m}, s_{3}, \ldots, s_{n-1}, s_{n}, s_{n-1}, \ldots, s_{3}, s_{2}\right)
$$

where $m=m\left(s_{1}, s_{2}\right)$. By reasoning similar to (4), $\mathbf{s}$ is a reduced word for some $w \in W^{\langle s\rangle}$ that is not fully commutative.

Since every maximal quotient of $W=A_{n}$ is minuscule, for the remainder of the proof we may assume that $W$ is not of type $A$, and hence also that $s$ is an end node of $\Gamma$.

(6) $\Gamma$ cannot have both a vertex of degree 3 and an edge of weight $\geq 4$. Otherwise, by following a path from $s$ we will reach either a vertex of degree 3 or an edge of weight $\geq$ 4. If the former occurs first, then by Lemma 6.3, we can reduce to a configuration of the type that was eliminated in (5). If the latter occurs, we can use Lemma 6.3 to reduce to a configuration of generators $s_{1}, \ldots, s_{n} \in S$ that induce a subgraph of $\Gamma$ isomorphic to the one in figure $3(\mathrm{~b})$, with $s=s_{1}$ and $m=m\left(s_{1}, s_{2}\right) \geq 4$. However in that case,

$$
\left(\left\langle s_{1}, s_{2}\right\rangle_{m}, s_{3}, \ldots, s_{n-2}, s_{n-1}, s_{n}, s_{n-2}, \ldots, s_{2}, s_{1}\right)
$$

is a suitable reduced word for some $w \in W^{\langle s\rangle}$ that is not fully commutative.

(7) $\Gamma$ has at most one edge of weight $\geq 4$. If $\Gamma$ has two or more edges of weight $\geq 4$, then $\Gamma$ must be a path and $s$ must be an end node, thanks to (5) and (6). By following the path from $s$, we can use Lemma 6.3 to reduce to the case of a path $s=s_{1}, s_{2}, \ldots, s_{n}$ in $\Gamma$ such that $m=m\left(s_{1}, s_{2}\right) \geq 4$ and $m\left(s_{n-1}, s_{n}\right) \geq 4$. However in that case,

$$
\left(\left\langle s_{1}, s_{2}\right\rangle_{m}, s_{3}, \ldots, s_{n-1}, s_{n}, s_{n-1}, \ldots, s_{3}, s_{2}, s_{1}\right)
$$

is a suitable reduced word for some $w \in W^{\langle s\rangle}$ that is not fully commutative.

In the following, we will continue to construct explicit reduced words for members of $W$ that are not fully commutative; however, in most of the remaining cases, the structure of the commutativity and braid equivalence classes are sufficiently complex that it is easier to deduce what is needed by examining heaps. More specifically, in (most of) the remaining constructions, we present a pair of heaps, and it is left to the reader to check the following: (i) Each heap has exactly one convex chain with alternating labels $i, j, i, \ldots$ of cardinality $m=m\left(s_{i}, s_{j}\right) \geq 3$ for some pair of generators $s_{i}, s_{j}$. (ii) The braid relation $\left\langle s_{i}, s_{j}\right\rangle_{m} \approx$ 

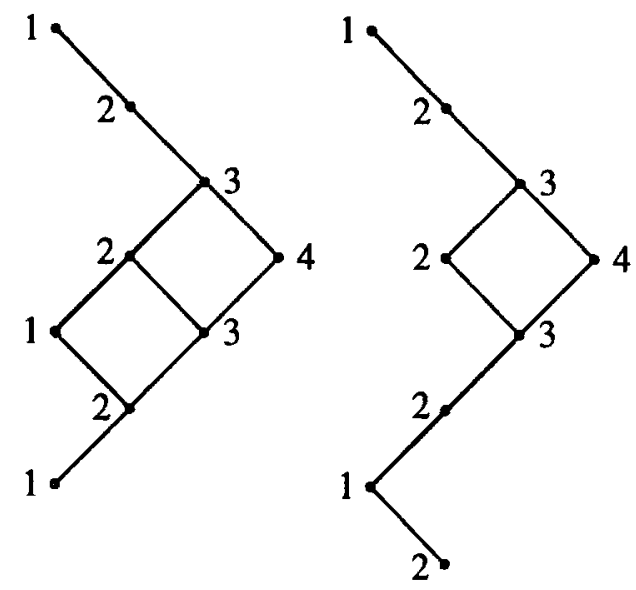

Figure 10.

$\left\langle s_{j}, s_{i}\right\rangle_{m}$, when applied to a linear extension of each heap, interchanges the two heaps. (iii) Both heaps have a maximum element, and the label of this element corresponds to $s$. These properties collectively imply that some $w \in W^{(s)}$ has exactly two commutativity classes and hence cannot be fully commutative.

(8) An edge of weight $\geq 4$ must be adjacent to an end node. Otherwise, by Lemma 6.3 we can reduce to a case in which there is a path $s=s_{1}, s_{2}, s_{3}, s_{4}$ with $m\left(s_{2}, s_{3}\right) \geq 4$. By (7), we may also assume that $m\left(s_{1}, s_{2}\right)=3$. However in that case, the pair of braid-related heaps in figure 10 prove the existence of some $w \in W^{\langle s\rangle}$ that is not fully commutative.

Suppose now that $\Gamma$ is a path, say $s_{1}, s_{2}, \ldots, s_{n}$. By (5), (7) and (8), we may assume that $m=m\left(s_{1}, s_{2}\right) \geq 4$, all other edge weights are 3 , and $s=s_{1}$ or $s=s_{n}$. If $n=2$ then $W$ is a dihedral group (a case covered by (c)), so assume $n \geq 3$. We may also assume $m \geq 5$, since otherwise $m=4, W=B_{n}$, and both end nodes correspond to minuscule quotients. If $s=s_{1}, m \geq 5$ and $n \geq 3$, then $w=s_{2} s_{3} s_{2} s_{1} s_{2} s_{1}$ is a member of $W^{\langle s\rangle}$ that is not fully commutative, so we can assume $s=s_{n}$. If $n=3$ and $m=5$, then $W=H_{3}$ and $W_{J}=I_{2}(5)$ (a case covered by (c)). If $n=3$ and $m \geq 6$, then $w=s_{2} s_{3} s_{2} s_{1} s_{2} s_{1} s_{2} s_{3}$ is a member of $W^{(s)}$ that is not fully commutative. With Lemma 6.3, this eliminates all cases with $n \geq 3$ and $m \geq 6$. Finally, if $n=4$ and $m=5$ (i.e., $W=H_{4}, W_{J}=H_{3}$ ), then the pair of braid-related heaps in figure 11 prove the existence of some $w \in W^{\langle s\rangle}$ that is not fully commutative. With Lemma 6.3, this eliminates all cases with $n \geq 4$ and $m=5$.

The only remaining possibility is that $W=Y(p, q, r)$ (see figure 4 ), and that $s$ is the end node of (say) the branch of length $p$. Continuing the labeling established in Section 5 , we let $s_{0}$ denote the vertex of degree 3 , and let $s_{1}, \ldots, s_{p} ; s_{1}^{\prime}, \ldots, s_{q}^{\prime} ; s_{1}^{\prime \prime}, \ldots, s_{r}^{\prime \prime}$ denote the generators along the three branches of $\Gamma$. If $q=r=1$, then $W=D_{n}$ and $W^{J}$ is minuscule. On the other hand, if $q, r \geq 2$, then the braid-related heaps in figure 12 provide a member of $W^{j}$ that is not fully commutative for the case $p=1$, and hence we may also 

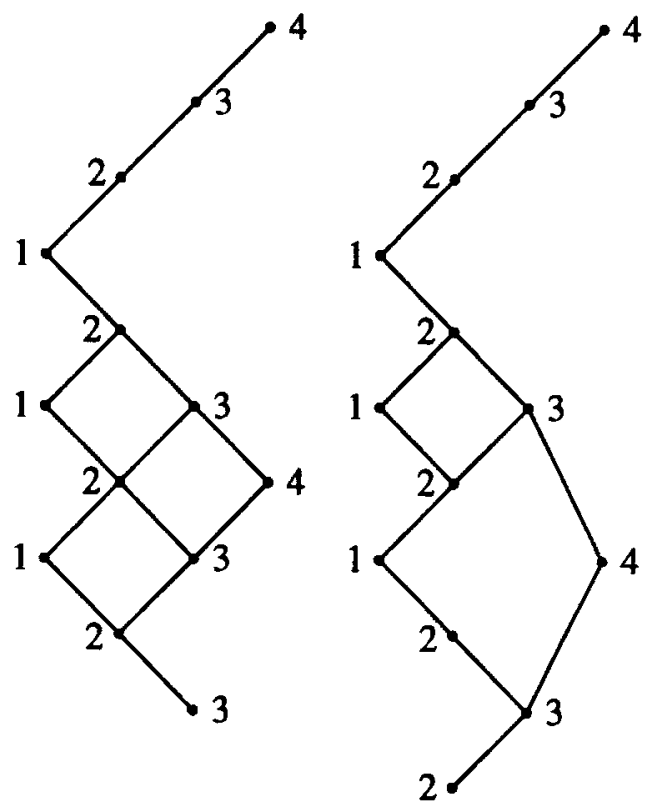

Figure 11.
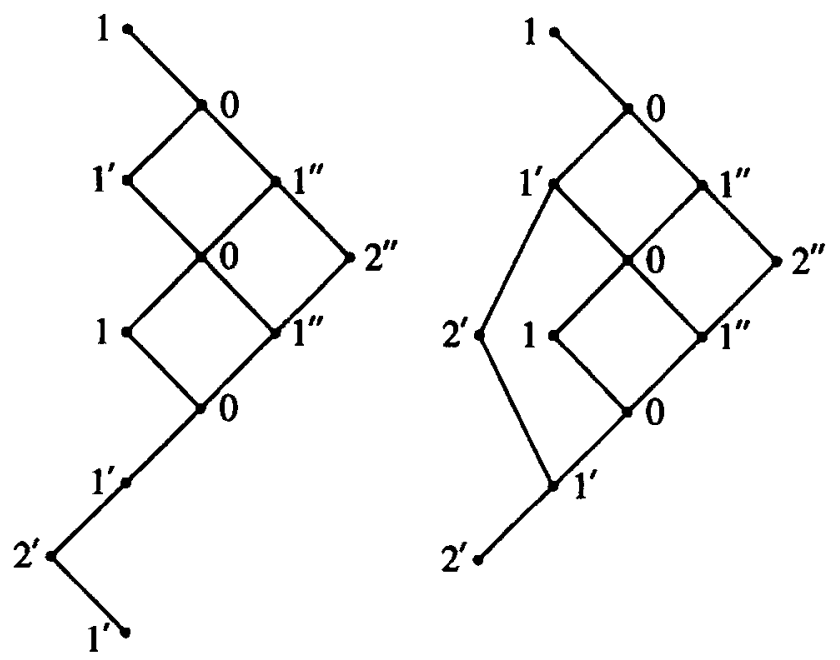

Figure 12. 


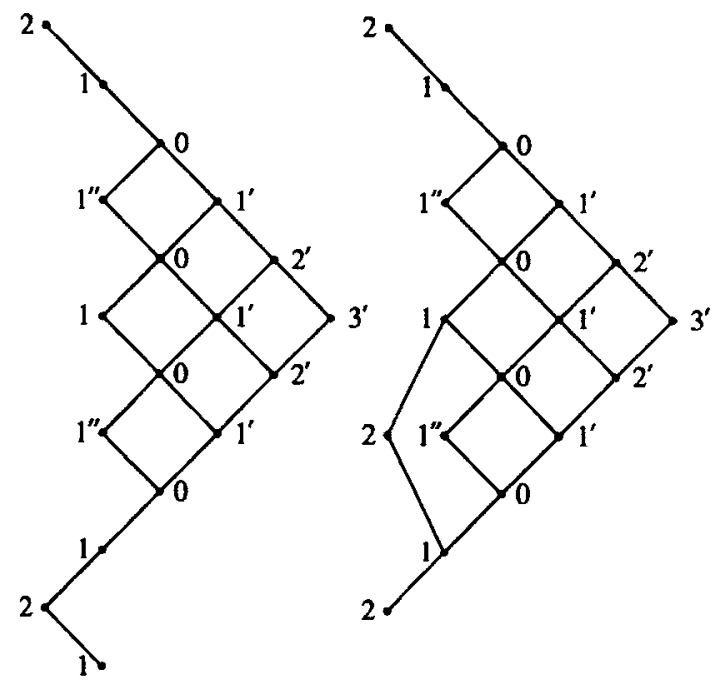

Figure 13.

eliminate $p>1$ (Lemma 6.3). For what remains, we may thus assume $q \geq 2$ and $r=1$. Now if $p=1$ then $W=D_{n}$ and $W^{J}$ is again minuscule, so we may further assume that $p \geq 2$. If $q \geq 3$, then the heaps in figure 13 provide a member of $W^{J}$ that is not fully commutative for the case $p=2$, and hence we may also eliminate $p \geq 3$ (again Lemma 6.3). Thus $q=2$. If $p=2$, then $W=E_{6}$ and $W_{J}=D_{5}$, and if $p=3$ then $W=E_{7}$ and $W_{J}=E_{6}$, both of which yield minuscule quotients. All that remains is $p \geq 4, q=2$, and $r=1$; however in that case, Lemma 6.3 and the heaps in figure 14 prove that $W^{J}$ has members that are not fully commutative.

\section{Consequences for the Bruhat order}

Let $T=\left\{w s w^{-1}: w \in W, s \in S\right\}$ denote the set of (abstract) reflections in $W$. The Bruhat order (e.g., $[3,9]$ ) may be defined as the partial ordering $\leq_{B}$ on $W$ generated by the transitive closure of the relations

$$
w<_{B} w t \quad \text { whenever } \ell(w)<\ell(w t)
$$

for all $w \in W, t \in T$. We note that there is no distinction between a "left" and "right" Bruhat order, since $t w=w\left(w^{-1} t w\right)>_{B} w$ if and only if $\ell(t w)>\ell(w)$. It is also clear that the Bruhat order refines both the left and right weak orders; i.e., $x \leq_{L R} y \Rightarrow x \leq_{B} y$ for all $x, y \in W$. However, unlike the weak ordering, the fully commutative part of $W$ and the parabolic quotients $W^{j}$ need not be order ideals of $\left(W, \leq_{B}\right)$.

In [11], Proctor classifies the parabolic quotients of finite Weyl groups whose Bruhat orderings are lattices; aside from the minuscule quotients, the only other examples occur in the case $W=G_{2}{ }^{3}$. Also, it is implicit in [11] and explicit for the symmetric group case in 


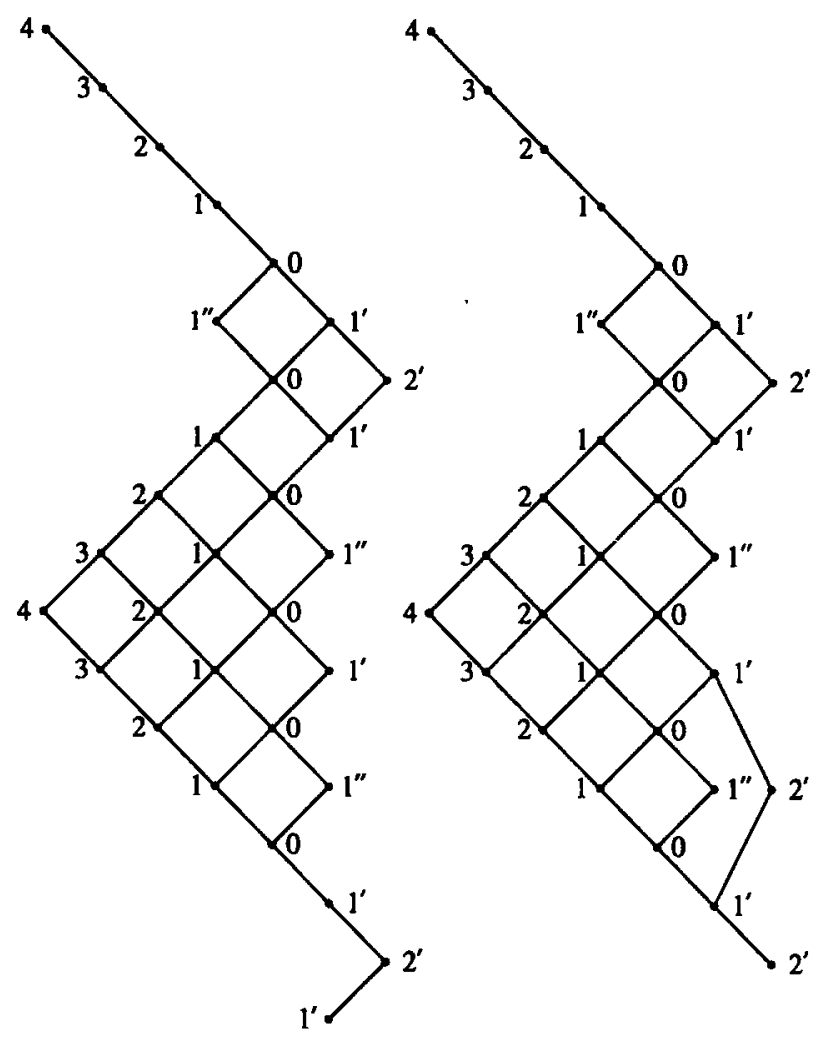

Figure 14.

([3] (4.9)) that the Bruhat ordering and weak ordering of a minuscule quotient are identical. The following result characterizes the parabolic quotients of arbitrary Coxeter groups with these properties.

Theorem 7.1 If $W$ is irreducible and $J$ is a proper subset of $S$, then the following are equivalent.

(a) $\left(W^{J}, \leq_{B}\right)$ is a lattice.

(b) $\left(W^{J}, \leq_{B}\right)$ is a distributive lattice.

(c) $\left(W^{J}, \leq_{L}\right)$ is a distributive lattice.

(d) $\left(W^{J}, \leq_{B}\right)=\left(W^{J}, \leq_{L}\right)$.

(e) $W^{J}$ is minuscule, or $\left(W, W_{J}\right) \cong\left(H_{3}, I_{2}(5)\right)$ or $\left(I_{2}(m), A_{1}\right)($ possibly $m=\infty)$.

Proof: First we show that each of the quotients listed in (e) satisfies properties (a)-(d). In case $W$ is a (possibly infinite) dihedral group and $J$ is a singleton, it is easy to check that $\left(W^{J}, \leq_{L}\right)$ is a total order. In particular, it is a distributive lattice. Since the Bruhat order refines the weak order, it follows that the two orders must coincide. Otherwise, in the remaining cases $W^{J}$ is a quotient of a finite group, and thus (Proposition 2.6) has a 
unique maximal element with respect to $\leq_{L}$. By Theorem 6.1, every member (including the top element) of $W^{J}$ is fully commutative, so by Theorem 3.2 it follows that $\left(W^{J}, \leq_{L}\right)$ is a distributive lattice. To prove that the remaining properties hold, it thus suffices to show that the Bruhat order and weak order coincide on $W^{J}$.

In case $W=H_{3}$ and $W_{J}=I_{2}(5)$, Theorem 3.2 implies that $\left(W^{J}, \leq_{L}\right)$ is isomorphic to the lattice of order ideals of the heap in figure 9. One can see directly that this lattice has exactly two incomparable elements; namely, $w_{1}=s_{1} s_{2} s_{1} s_{2} s_{3}$ and $w_{2}=s_{3} s_{2} s_{1} s_{2} s_{3}$ (using the labels for generators introduced in Section 6). Since these two elements have the same length, they must also be incomparable in the Bruhat order. Thus the two orders coincide in this case.

Now consider the minuscule case. Continuing our previous notation, let $\mathbf{E}, \Delta$, and $\Phi$ be as they were defined in Section 6 , and let $\lambda$ be a minuscule weight with stabilizer $W_{J}$. Define $r$ to be the linear functional on $\mathbf{E}$ satisfying $r(\alpha)=1$ for all $\alpha \in \Delta$.

Lemma 7.2 For $w \in W^{J}$, we have $\ell(w)=r(\lambda)-r(w \lambda)$.

Proof: The case $\ell(w)=0$ is obvious, so assume $\ell(w) \geq 1$ and choose $\alpha \in \Delta$ so that $s_{\alpha} w<_{L} w$. By Lemma 6.2, it follows that $(w \lambda, \alpha)<0$, so by Exercise VI.1.24 in [4], we have $\left(w \lambda, \alpha^{\vee}\right)=-1$. Hence $s_{\alpha}(w \lambda)=w \lambda+\alpha$, so $r\left(s_{\alpha} w \lambda\right)=r(w \lambda)+1$ and the result follows by induction with respect to $\ell(w)$.

Now let $t \in T, w \in W$ be such that $t w<_{B} w$ is a covering relation. We have $t=s_{\alpha}$ for some (positive) $\alpha \in \Phi$. It is necessary that $t w \lambda \neq w \lambda$; otherwise, $t w$ would be a shorter member of the coset containing $w$. Therefore $(w \lambda, \alpha) \neq 0$, and hence by the Bourbaki exercise (ibid.), $\left(w \lambda, \alpha^{\vee}\right)= \pm 1$. It follows that $t w \lambda=w \lambda \pm \alpha$, so $r(\alpha)=\ell(w)-\ell(t w)$, by Lemma 7.2. However, the Bruhat order is graded by the length function (e.g., [9], Section 5.11), so the only covering relations involve pairs with a length difference of one. It follows that $r(\alpha)=1$, so $\alpha$ is a simple root and $t w<_{L} w$. Thus every covering relation of the Bruhat order is also a covering relation of the weak order, so the two coincide.

Turning to the converse, we show that if $W^{J}$ is any of the quotients not listed in (e), then none of the properties (a)-(d) hold. By Theorem 6.1, there are two possibilities: either $W^{J}$ contains elements that are not fully commutative, or $W$ has rank $\geq 3$ and every edge-weight of $\Gamma$ is infinite.

Suppose that $w \in W^{J}$ is not fully commutative. By replacing $w$ with some $w^{\prime}<_{L} w$ if necessary, we can assume that there is a reduced expression of the form $w=x_{0} y$, where $x_{0}$ is the element of length $m(s, t)$ in the parabolic subgroup generated by some $\{s, t\} \subset S$ such that $m(s, t) \geq 3$. Consider the subinterval of $\left(W^{J}, \leq_{L}\right)$ from $y$ to $w$. By Proposition 2.3, this interval is order-isomorphic to the weak ordering of the parabolic subgroup generated by $\{s, t\}$ (cf. figure 1(a)). This interval is not a distributive lattice, so (c) does not hold. (This fact is also an immediate consequence of Theorem 3.2, given that $W^{J}$ contains elements that are not fully commutative.) Furthermore, with respect to the Bruhat order, this interval contains additional relations, such as $s y<_{B}$ sty and $t y<_{B} t s y$ (cf. figure 1(b)), so (d) does not hold. Since we also have $s y<_{B} t s y$ and $t y<_{B} s t y$, it follows that $s y$ and $t y$ have no least upper bound relative to the Bruhat order, so and (a) and (b) do not hold. 
The remaining possibility is that $W$ has rank $\geq 3$ and every edge of $\Gamma$ has infinite weight. It follows that there must exist generators $s, t, u \in S$ such that $s \notin J$, and either (1) $m(s, t)=m(s, u)=\infty$ or (2) $m(s, t)=m(t, u)=\infty$. In case (1), consider the elements uts, sts, suts, and usts. It is easy to see that every reduced word for these elements ends with $s$, so they all belong to $W^{J}$. Secondly, it is not hard to show, using the subword property (e.g., [9], Section 5.10) or otherwise, that the two elements of length four are both upper bounds for the two elements of length three with respect to the Bruhat order, so (a) and (b) do not hold. The fact that sts and suts are unrelated with respect to the weak order shows that (d) does not hold. We also claim that the elements sts and $u t s$ have no upper bounds relative to $\leq_{L}$. By definition, the upper bounds for $s t s$ are the reduced expressions of the form $w s t s$. Since the only braid relations in $W$ involve pairs of commuting generators, it follows that every reduced word for wsts must have at least two occurrences of $s$ following any occurrence of $u$. In particular, no such reduced word can end with $(u, t, s)$, so wsts cannot be an upper bound for $u t s$. Hence $\left(W^{J}, \leq_{L}\right)$ is not a lattice and (c) fails.

In case (2), consider the elements tuts, suts, tsuts, and stuts. Again, it is easy to see that every reduced word for these elements ends with $s$, so they all belong to $W^{J}$. Using the subword property, one sees that both elements of length five are upper bounds for both elements of length four with respect to the Bruhat order, so (a) and (b) fail. Also, tuts and tsuts are unrelated with respect to $\leq_{L}$, so (d) fails. Finally, we argue that tuts and suts have no upper bounds relative to $\leq_{L}$. Indeed, if $x=w s u t s$ is reduced, then every reduced word for $x$ must have exactly one $t$ occurring between the last two occurrences of $s$. In particular, no such reduced word can end with $(t, u, t, s)$, so $x$ cannot be an upper bound for tuts. Hence $\left(W^{J}, \leq_{L}\right)$ is not a lattice and (c) fails.

\section{Notes}

1. A Coxeter group is simply-laced if the product of any pair of noncommuting generators has order 3 .

2. We thank R. Proctor for suggesting this terminology.

3. However in [11], Proctor also remarks without proof that among the finite Coxeter groups, the Bruhat order on $W^{J}$ with $\left(W, W_{J}\right) \cong\left(H_{3}, A_{2}\right)$ is a lattice. Theorem 7.1 shows that this is incorrect.

\section{References}

1. S. Billey and M. Haiman, "Schubert polynomials for the classical groups," J. Amer. Math. Soc. 8 (1995), 443-482.

2. S. Billey, W. Jockusch, and R. Stanley, "Some combinatorial properties of Schubert polynomials," J. Alg. Combin. 2 (1993), 345-374.

3. A. Björner, "Orderings of Coxeter groups," Contemporary Math. 34 (1984), 175-195.

4. N. Bourbaki, Groupes et Algèbres de Lie, Chaps. IV-VI, Masson, Paris, 1981.

5. P. Cartier and D. Foata, Problèmes Combinatoires de Commutation et Réarrangements, Lect. Notes in Math. Vol. 85, Springer-Verlag, 1969.

6. C.K. Fan, A Hecke Algebra Quotient and Properties of Commutative Elements of a Weyl Group, Ph.D. thesis, MIT, 1995.

7. C.K. Fan, "A Hecke algebra quotient and some combinatorial applications," J. Alg. Combin., to appear. 
8. S.V. Fomin and A.N. Kirillov, "Combinatorial $B_{n}$-analogues of Schubert polynomials," Trans. Amer. Math. Soc., to appear.

9. J.E. Humphreys, Reflection Groups and Coxeter Groups, Cambridge Univ. Press, Cambridge, 1990.

10. T.K. Lam, B and D Analogues of Stable Schubert Polynomials and Related Insertion Algorithms, Ph.D thesis, MIT, 1995.

11. R.A. Proctor, "Bruhat lattices, plane partition generating functions, and minuscule representations," Europ. J. Combin. 5 (1984), 331-350.

12. R.P. Stanley, Enumerative Combinatorics, Vol. I, Wadsworth \& Brooks/Cole, Monterey, 1986.

13. J.R. Stembridge, "Some combinatorial aspects of reduced words in finite Coxeter groups," Trans. Amer. Math. Soc., to appear.

14. G.X. Viennot, "Heaps of pieces I: Basic definitions and combinatorial lemmas," in Combinatoure Énumératue, G. Labelle and P. Leroux (Eds.), pp. 321-350, Lect. Notes in Math. Vol. 1234, Springer-Verlag, 1985. 